Preprints of the

Max Planck Institute for

Research on Collective Goods

Bonn 2017/2

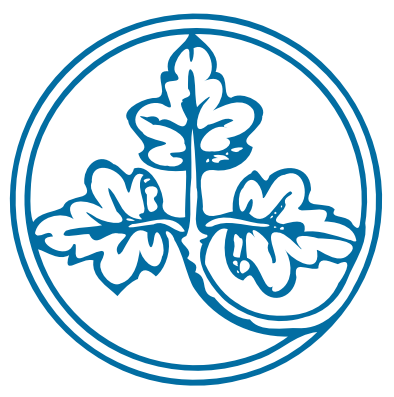

The Generic Possibility of Full Surplus Extraction in Models with Large Type Spaces

Alia Gizatulina

Martin Hellwig

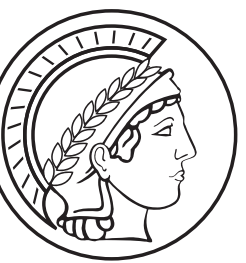




\title{
The Generic Possibility of Full Surplus Extraction in Models with Large Type Spaces
}

\author{
Alia Gizatulina / Martin Hellwig
}

February 2017

This version: May 25, 2017 


\title{
The Generic Possibility of Full Surplus Extraction in Models with Large Type Spaces*
}

\author{
Alia Gizatulina $^{\dagger}$ and Martin Hellwig ${ }^{\ddagger}$
}

This version: May 25, 2017

\begin{abstract}
McAfee and Reny (1992) have given a necessary and sufficient condition for full surplus extraction in naive type spaces with a continuum of payoff types. We generalize their characterization to arbitrary abstract type spaces and to the universal type space and show that in each setting, full surplus extraction is generically possible. We interpret the McAfee-Reny condition as a much stronger version of injectiveness of belief functions and prove genericity by arguments similar to those used to prove the classical embedding theorem for continuous functions. Our results can be used to also establish the genericity of common priors that admit full surplus extraction.
\end{abstract}

JEL Classification: D40, D44, D80, D82

Keywords: mechanism design, surplus extraction,abstract type spaces, universal type space, genericity, correlated values, correlated information, strategic continuity.

*This paper supersedes our earlier paper "On the Genericity of the McAfee-Reny Condition for Full Surplus Extraction in Models with a Continuum of Types", Preprint 08/2015, Max Planck Institute for Research on Collective Goods, Bonn. For helpful discussions, comments, and suggestions, we are grateful to an associate editor and two referees as well as Dirk Bergemann, Yi-Chun Chen, Olga Gorelkina, Alfredo di Tillio and the participants of the 2013 EEA-ESEM conference and of the 2013 Bonn Workshop on Advances in Mechanism Design.

${ }^{\dagger}$ alia.gizatulina@unisg.ch, University of St. Gallen, Varnbüelstr. 19, 9000 St. Gallen, Switzerland

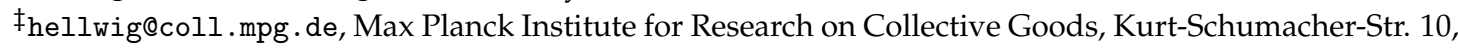
53113 Bonn, Germany 


\section{Introduction}

A central theme in the economics of information concerns the ability of agents to earn rents because they have private information. For example, the buyer of a good may be able to obtain a surplus because the seller does not know how much the good is worth to the buyer.

However, Crémer and McLean (1988) have shown that, when there are multiple potential buyers for a good and these buyers have quasilinear utility functions with correlated private values, then, under certain conditions, in a Bayesian setting, a seller can extract all the surplus from the sale of his good, i.e. all information rents can be made to disappear. Specifically, if the potential buyers have only finitely many types, a Bayesian incentive mechanism that extracts all the potentially available surplus from buyers can be designed if and only if, for each agent $i$ and each type $t_{i}$ of this agent, the vector of probabilities that agent $i$ assigns to different constellations of the other agents' types when his own type is $t_{i}$ cannot be represented as a convex combination of the vectors of beliefs that he has at types other than $t_{i}$.

McAfee and Reny (1992) extended the analysis of Crémer and McLean (1988) to the case where each agent's type set is the unit interval and where each agent's beliefs about other agents' types are given by a probability distribution with a continuous density function. They showed that (approximately) full surplus extraction can be obtained if and only if the density functions that represent agents' beliefs satisfy a function space version of the Crémer-McLean condition.

Our paper makes three contributions to this literature. First, we extend the analysis of McAfee and Reny (1992) to allow for arbitrary abstract (Harsanyi) type spaces, rather than naive type spaces in which "types" and payoff parameters are the same so that beliefs depend only on payoff parameters. We give a necessary and sufficient condition for full surplus extraction in an arbitrary abstract type space (with arbitrary beliefs) and call it the generalized McAfee-Reny condition. This condition coincides with the McAfee-Reny condition if the mapping from abstract types to payoff parameters and beliefs is injective, but otherwise it is slightly weaker.

Second, we show that full surplus extraction is generic in the sense that, for a given type space $T_{i}$ of agent $i$, the generalized McAfee-Reny condition holds for a residual set, i.e., for a countable intersection of open and dense sets, of continuous functions mapping types into payoff parameters and beliefs. For models with a continuum of types, generic- 
ity of full surplus extraction has been a matter of dispute. ${ }^{1}$ On the one hand, Heifetz and Neeman (2006) have suggested that full surplus extraction is generically impossible. ${ }^{2}$ On the other hand, Chen and Xiong (2013) have shown that in a particular class of models, approximately full surplus extraction is generically possible. Our result is both stronger and more general than that of Chen and Xiong (2013). It is also "topology-free" in the sense that we do not specify a topology on beliefs but only require the topology on beliefs to by induced by a metric that is a convex function.

We use ideas from embedding theory. An embedding is a continuous injective function from a space $X$ to a space $Y$. The classical embedding theorem asserts that, if $X$ is a compact finite-dimensional metric space and $Y$ is a metric space with a sufficiently high dimension, the set of embeddings is residual in the space of continuous functions from $X$ to $Y$, endowed with the uniform topology. ${ }^{3}$ The McAfee-Reny condition for surplus extraction is similar to, but substantially stronger than injectiveness. Therefore we cannot use the embedding theorem itself but need a new mathematical result. The proof of this result is similar to the proof of the embedding theorem but makes essential use of the fact that, in a model with a continuum of types, the space of beliefs, i.e. probability measures over constellations of the other agents' types, is infinite-dimensional.

The third contribution of this paper extends our analysis to the universal type space, i.e. the space that is obtained if agents' "types" are defined by their payoff parameters and their hierarchies of beliefs about other agents' payoff parameters, beliefs about other agents' payoffs and first-order beliefs, beliefs about other agents' payoffs, first-order and second-order beliefs... etc. In this setting, an agent's belief hierarchy determines a probability measure over the possible constellations of the other agents' payoff parameters and belief hierarchies. The induced mapping from "types" of agent $i$ to beliefs over the other agent's "types" violates the generalized McAfee-Reny condition if the domain is taken to be the universal types space as a whole, but the restriction of this mapping to a sub-

\footnotetext{
${ }^{1}$ For models with finitely many types, as in Crémer and McLean (1988), genericity of full surplus extraction is automatically obtained if the set of other agents' types is sufficiently large.

${ }^{2}$ Barelli (2009) also makes this claim, but Chen and Xiong (2011) show that his analysis involves an error.

${ }^{3}$ In Gizatulina and Hellwig (2014), we used this theorem to show that injective belief functions are generic in the space of continuous functions from agents' types to their beliefs. If a belief function is injective, then, regardless of how the agent's payoff parameters depend on his type, his payoff parameters can be inferred from his beliefs. As was shown by Neeman (2004) and Heifetz and Neeman (2006), this so-called BDP property ("beliefs determine preferences") is necessary for full surplus extraction; see also Section 2.1 below. The relation between Heifetz and Neeman (2006) and our work is further discussed in Section 4.1 below.
} 
set of the universal type space may satisfy this condition. We show that the generalized McAfee-Reny condition holds on a given subset of the universal type space if and only if it holds for all abstract type spaces that generate constellations of payoff parameters and belief hierarchies in the given subset of the universal type space. We also show that the set of compact subsets of the universal type space that satisfy the generalized McAfee-Reny condition is a residual subset of the set of all compact subsets of the universal type space.

Genericity results are highly sensitive to the topologies that are used. As indicated above, we do not actually specify any particular topologies but give qualitative conditions on the topologies. These conditions are met by all the topologies that have been proposed in the recent literature. ${ }^{4}$ For example, our genericity result for full surplus extraction in the universal type space holds regardless of whether the universal type space is given the product topology, as in Mertens and Zamir (1985), or the uniform strategic topology, as in Dekel, Fudenberg, and Morris (2006).

Our results contrast with the suggestion of Heifetz and Neeman (2006) that in arbitrary infinite type spaces full surplus extraction will only be possible in exceptional cases. The difference between their analysis and ours is explained in detail in Section 4.1 below. Despite the difference between their assessment of genericity and ours, we do not disagree with their assessment that full surplus extraction is unlikely in the real world. However, in our view, the inability to extract surplus is due to the mechanism designer's lack of information about the participants' belief mappings, rather than the exceptional nature of belief mappings supporting full surplus extraction. In any given situation, it seems quite unlikely that a mechanism designer should know the participants' belief functions as precisely as he must in order to fully exploit the dependence of beliefs on types for surplus extraction. This lack of information about the participants' belief functions should be dealt with on its own terms, for example by a robustness requirement along the lines of Bergemann and Morris (2005).

As we already mentioned, Chen and Xiong (2013) also have a result showing that full surplus extraction is generically possible. They use a very different approach, defining the FSE property as a property of priors and relying on approximations by finite type spaces, in which the Crémer-McLean result applies. Their approach works for allocation problems and payment functions that allow for the exclusion of individuals but cannot be used if exclusion is not feasible, for example if the allocation problem involves the

\footnotetext{
${ }^{4}$ For abstract type spaces, see Engl (1995), for the universal type space, see, in particular, Dekel, Fudenberg, and Morris (2006), and Chen, DiTillio, Faingold, and Xiong (2010).
} 
provision of a non-excludable public good. Their approach also cannot be used if the space of beliefs over constellations of other agents' types has a topology under which the measures with finite supports are not dense, such as the topology of weak convergence that is induced if the spaces of the other agents' types have the uniform strategic topology of Dekel, Fudenberg, and Morris (2006) and Chen, DiTillio, Faingold, and Xiong (2010).

In the following, Section 2 presents our results for abstract type space. Sections 2.1 and 2.2 introduce the McAfee-Reny condition and the generalized McAfee-Reny condition for full surplus extraction in naive type spaces and in arbitrary abstract type spaces. Section 2.3 presents the basic genericity results for these conditions. Section 2.4 provides a genericity result for common priors generating belief functions that admit full surplus extraction. Section 2.5 proves the main genericity theorem.

Section 3 presents our results for the universal type space. Following an introduction of the universal type space in Section 3.1, Section 3.2 discusses the feasibility of full surplus extraction as a property of compact subsets of the universal type space. Section 3.3 gives a genericity result for compact subsets that admit full surplus extraction, Section $3.4 \mathrm{a}$ genericity result for common priors on the universal type space.

Section 4 relates our analysis to that of Heifetz and Neeman (2006), and Chen and Xiong (2013). Section 5 contains some concluding remarks.

\section{Full Surplus Extraction in Abstract-Type-Space Models}

\subsection{The McAfee-Reny Condition}

McAfee and Reny (1992) consider the following problem: Suppose that a game of incomplete information between agents $i=1, \ldots, I$ has a Bayes-Nash equilibrium in which agent $i$ obtains the payoff

$$
\pi_{i}\left(t_{1}, \ldots, t_{I}\right)
$$

where $t_{1}, \ldots, t_{I}$ are different agents' types. Is it possible to design incentive-compatible systems of participation fees that extract this surplus from each agent? ${ }^{5}$

\footnotetext{
${ }^{5}$ In Crémer and McLean (1988) and McAfee and Reny (1992), the function $\pi_{i}$ indicates the equilibrium payoff from the truth-telling equilibrium of a second-price auction. As was pointed out to us by a referee, there is no need to restrict the analysis to payoff functions resulting from dominant-strategy equilibria. It is however necessary to assume that $\pi_{i}$ is continuous.
} 
For any $i$, the type $t_{i}$ of agent $i$ is an element of a metric space $T_{i}$. Given the type $t_{i}$, the belief of agent $i$ if given by a probability measure $b_{i}\left(t_{i}\right)$ about the other agents' types. The belief $b_{i}\left(t_{i}\right)$ is an element of the space $\mathcal{M}\left(T_{-i}\right)$ of probability measures on the product space $T_{-i}:=\prod_{j \neq i} T_{j}$. Given $b_{i}\left(t_{i}\right)$, the agent's expected payoff from participating in the game with the equilibrium payoff function $\pi_{i}$ is given as

$$
\bar{\pi}_{i}\left(t_{i}\right):=\int_{T_{-i}} \pi_{i}\left(t_{1}, \ldots, t_{I}\right) b_{i}\left(d t_{-i} \mid t_{i}\right) .
$$

McAfee and Reny (1992) consider a system of participation fees with the following structure. Each agent $i$ can choose one out of $N_{i}$ fee schedules $z_{1}^{i}, \ldots, z_{N_{i}}^{i}$, which make the fee he has to pay depend on the other agents' types. Thus, if agent $i$ chooses the schedule $z_{n}^{i}$, his payment will be $z_{n}^{i}\left(t_{-i}\right)$. Given his type $t_{i}$ and his belief $b_{i}\left(t_{i}\right)$, his expected payment under the fee schedule $z_{n}$ is

$$
\bar{z}_{n}^{i}\left(b_{i}\left(t_{i}\right)\right):=\int_{T_{-i}} z_{n}^{i}\left(t_{-i}\right) b_{i}\left(d t_{-i} \mid t_{i}\right)
$$

and the agent may be presumed to choose the schedule with the smallest expected payment $\bar{z}_{n}\left(t_{i}\right)$. His actual expected payment is thus equal to

$$
\bar{Z}_{i}\left(b_{i}\left(t_{i}\right)\right):=\min \left(\bar{z}_{1}^{i}\left(b_{i}\left(t_{i}\right)\right), \ldots, \bar{z}_{N_{i}}^{i}\left(b_{i}\left(t_{i}\right)\right)\right) .
$$

The belief function $b_{i}: T_{i} \rightarrow \mathcal{M}\left(T_{-i}\right)$ is said to admit full surplus extraction in the sense of McAfee and Reny (1992) if and only if, for every continuous function $\bar{\pi}_{i}: T_{i} \rightarrow \mathbb{R}_{+}$and every $\varepsilon>0$, there exists a system $z_{1}^{i}, \ldots, z_{N_{i}}^{i}$ of participation fee schedules for agent $i$ such that the induced expected payment $\bar{Z}_{i}\left(b_{i}\left(t_{i}\right)\right)$ as given by (3) and (4) satisfies

$$
\bar{\pi}_{i}\left(t_{i}\right)-\varepsilon \leq \bar{Z}_{i}\left(b_{i}\left(t_{i}\right)\right) \leq \bar{\pi}_{i}\left(t_{i}\right)
$$

for all $t_{i} \in T_{i}{ }^{6}$ Whereas the surplus that can be extracted from agent $i$ depends on the

\footnotetext{
${ }^{6}$ Condition (5) provides for approximate rather than full surplus extraction. As explained by McAfee and Reny, exact surplus extraction is not to be expected. For example, if the belief function $b_{i}$ has a continuous density, one can find an expected-payoff function $\bar{\pi}_{i}$ for which exact surplus extraction is not possible, i.e. there is no system of fee schedules $z_{1}^{i}, \ldots, z_{N_{i}}^{i}$ such that $\min \left(z_{1}^{i}\left(t_{i}\right), \ldots, \bar{z}_{N_{i}}^{i}\left(t_{i}\right)\right)=\bar{\pi}_{i}\left(t_{i}\right)$ for all $t_{i}$. However, since the choice of $\varepsilon$ in (5) is arbitrary, the divergence from full surplus extractions can be made arbitrarily small. Chen and Xiong (2013) have a different and somewhat weaker notion of approximate, rather than full surplus extraction. Whereas McAfee and Reny (1992) define the FSE property type by type, Chen and Xiong (2013) define the FSE property in terms of ex ante expected surplus, as a property of priors assigning small probabilities to the set of types for which the unrealized or unextracted surplus is significant.
} 
functions $b_{i}$ and $\bar{\pi}_{i}$ jointly, the FSE property requires full surplus extraction for all continuous $\bar{\pi}_{i}$ and therefore pertains to the belief function only.

McAfee and Reny (1992) give a necessary and sufficient condition under which a belief function has the FSE property. The following theorem extends their result to the present, more general formulation. ${ }^{7}$

THEOREM 2.1 Assume that $T_{i}$ is compact. Assume also that the belief function $b_{i}$ maps $T_{i}$ continuously into $\mathcal{M}\left(T_{-i}\right)$ where $\mathcal{M}\left(T_{-i}\right)$ is endowed with a topology that is at least as fine as the topology of weak convergence of probability measures. Then $b_{i}$ admits full surplus extraction if and only if, for every $\bar{t}_{i} \in T_{i}$ and every probability measure $\mu_{i}$ on $T_{i}$,

$$
\varphi_{b_{i}}\left(\mu_{i}\right)=\varphi_{b_{i}}\left(\delta_{\bar{t}_{i}}\right) \text { implies } \mu_{i}=\delta_{\bar{t}_{i}}
$$

where $\delta_{\bar{t}_{i}}$ is the degenerate measure that assigns all mass to the the singleton $\left\{\bar{t}_{i}\right\}$ and $\varphi_{b_{i}}\left(\mu_{i}\right)$ is defined so that

$$
\varphi_{b_{i}}\left(B \mid \mu_{i}\right):=\int_{T_{i}} b_{i}\left(B \mid t_{i}\right) \mu_{i}\left(d t_{i}\right)
$$

for any measurable set $B \subset T_{-i}$.

The proof of Theorem 2.1 is sketched in the appendix. The argument is by and large the same as in McAfee and Reny (1992).

The McAfee-Reny condition $\left(^{*}\right)$ can be interpreted as an extended screening condition. For any belief function $b_{i}$ and any measure $\mu_{i} \in \mathcal{M}\left(T_{-i}\right)$, the measure $\varphi_{b_{i}}\left(\mu_{i}\right)$ indicates the beliefs that agent $i$ would have about the other agents' types if he thought that his own type was distributed as $\mu_{i}$. Given the assumption that each agent knows his own type, the notion of agent $i$ 's forming beliefs about the other agents' types on the basis that his own type is distributed as $\mu$ may seem strange This notion is natural though if we replace the type space $T_{i}$ by the space $\mathcal{M}\left(T_{i}\right)$ of probability measures on $T_{i}$ and if we think about an extended type of agent $i$ as measure $\mu_{i}$ on $T_{i}$. If the measure $\mu_{i}$ is nondegenerate, the agent knows his extended type but has only probabilistic beliefs about his own type (and by implication, his payoff type and his belief type).

\footnotetext{
${ }^{7}$ The result in McAfee and Reny (1992) actually assumes that $T_{i}$ is the unit interval and that, for any $t_{i} \in T_{i}$, the belief $b_{i}\left(t_{i}\right)$ has a density function $f_{b_{i}}: T_{i} \times T_{-i} \rightarrow \mathbb{R}_{+}$that is jointly continuous in $t_{i}$ and $t_{-i}$. However, McAfee and Reny (1992) also note, without proof, that their result holds whenever $T_{i}$ is a compact metric space.
} 
The McAfee-Reny condition is then equivalent to the requirement that the beliefs that are associated with any degenerate extended type, i.e. any measure on $T_{i}$ that is concentrated at a singleton, must be distinguishable from the beliefs that are associated with any other extended type. This requirement is stronger than injectiveness of the belief function on the original type space $T_{i}$ but weaker than injectiveness of the belief function on the extended type space $\mathcal{M}\left(T_{i}\right)$; it does not exclude the possibility that the same beliefs might be associated with two nondegenerate extended types. ${ }^{8}$

\subsection{The Generalized McAfee-Reny Condition}

We now turn to more general incomplete-information models of the form

$$
\mathcal{T}=\left\{T_{i}, \theta_{i}(.), b_{i}(.)\right\}_{i=1}^{I}
$$

where, for any $i, T_{i}$ is an abstract (Harsanyi) type space, $\Theta_{i}$ is a metric space, the set of payoff parameters for agent $i, \theta_{i}: T_{i} \rightarrow \Theta_{i}$, is a function that indicates how the payoff parameters of agent $i$ depend on the abstract type $t_{i}$, and $b_{i}: T_{i} \rightarrow \mathcal{M}\left(T_{-i}\right)$ is the agent's belief function.

The McAfee-Reny model of the preceding section corresponds to the special case where payoff parameter and abstract types coincide, i.e. where $\Theta_{i}=T_{i}$ and $\theta_{i}($.$) is the$ identity mapping. In this special case, beliefs depend only on payoff parameters.

In the more general formulation, equilibrium payoffs from a game would take the form

$$
\pi_{i}\left(t_{1}, \ldots, t_{I}\right)=\pi_{i}^{*}\left(\theta_{1}\left(t_{1}\right), \ldots, \theta_{I}\left(t_{I}\right), b_{1}\left(t_{1}\right), \ldots b_{I}\left(t_{I}\right)\right),
$$

and the expected payoff of agent $i$ with belief $b_{i}\left(t_{i}\right)$ would take the form

$$
\bar{\pi}_{i}\left(t_{i}\right)=\bar{\pi}_{i}^{*}\left(\theta_{i}\left(t_{i}\right), b_{i}\left(t_{i}\right)\right),
$$

\footnotetext{
${ }^{8}$ McAfee and Reny (1992) themselves give an interpretation of $\left(^{*}\right)$ that is based on the notion that $\mu$ can be thought of as a prior on $T_{i}$. In this case, a violation of $\left(^{*}\right)$ would indicate that, relative to the prior $\mu$, agent $i$ 's learning that his own type is $t_{i}$ does not provide him with any new information about the other agents' types. This explanation however is problematic if the measure $\mu$ does not have $t_{i}$ in its support, for example, if $\mu$ is a degenerate measure that assigns all mass to $t_{i}^{\prime} \neq t_{i}$. Moreover, the interpretation of $\mu$ as a prior raises the question of how to interpret the requirement that condition ${ }^{*}$ ) hold for all measures other than the degenerate measure with unit mass at $t_{i}$.
} 
so that the abstract type $t_{i}$ of agent $i$ affects the agent's expected payoff $\bar{\pi}_{i}\left(t_{i}\right)$ only through its impact on the payoff parameter $\theta_{i}\left(t_{i}\right)$ and the belief $b_{i}\left(t_{i}\right){ }^{9}$

If the functions $\bar{\pi}_{i}^{*}, \theta_{i}$, and $b_{i}$ are continuous, then obviously the expected-payoff function $\bar{\pi}_{i}$ in (9) belongs to the class of functions considered in the McAfee-Reny definition of the FSE property, By Theorem 2.1 therefore, (approximately) full surplus extraction from agent $i$ is still possible if the belief function $b_{i}$ satisfies the McAfee-Reny condition.

However, if all payoff functions take the form (8), a definition of the FSE property that requires surplus extraction for all payoff functions of the form (1), is too strong. ${ }^{10}$ We therefore introduce a less demanding condition and refer to it as the FSE* property. A pair $\left(\theta_{i}(\cdot), b_{i}(\cdot)\right)$ of functions relating abstract types to payoff parameters and beliefs has the FSE $^{*}$ property if and only if, for every continuous function $\bar{\pi}_{i}^{*}: \Theta_{i} \times \mathcal{M}\left(T_{-i}\right) \rightarrow \mathbb{R}_{+}$and every $\varepsilon>0$, there exists a system $z_{1}^{i}, \ldots, z_{N_{i}}^{i}$ of participation fee schedules for agent $i$ such that the induced expected payment $\bar{Z}_{i}\left(b_{i}\left(t_{i}\right)\right)$ as given by (3) and (4) satisfies

$$
\bar{\pi}_{i}^{*}\left(\theta_{i}\left(t_{i}\right), b_{i}\left(t_{i}\right)\right)-\varepsilon \leq \bar{Z}_{i}\left(b_{i}\left(t_{i}\right)\right) \leq \bar{\pi}_{i}^{*}\left(\theta_{i}\left(t_{i}\right), b_{i}\left(t_{i}\right)\right)
$$

for all $t_{i} \in T_{i}{ }^{11}$

Trivially, a pair $\left(\theta_{i}(\cdot), b_{i}(\cdot)\right)$ has the FSE* property if $b_{i}(\cdot)$ has the FSE property. The converse is true if and only if the map

$$
t_{i} \rightarrow\left(\theta_{i}\left(t_{i}\right), b_{i}\left(t_{i}\right)\right)
$$

is injective, or, equivalently, if the type space $T_{i}$ is non-redundant, i.e. no two distinct types generate the same pair of payoff parameters and beliefs.

The following result adapts Theorem 2.1 to the present, more general setting.

\footnotetext{
${ }^{9}$ Typically the payoff $\pi_{i}\left(t_{1}, \ldots, t_{I}\right)$ and expected payoff $\bar{\pi}_{i}\left(t_{i}\right)$ also depend on the functions $\theta_{i}(\cdot)$ and $b_{i}(\cdot)$ as these functions determine the participants' choices of strategies in whatever game they are playing. However this dependence does not matter for the possibility of surplus extraction. Therefore we do not make it explicit.

${ }^{10}$ For example, if $T_{i}=[0,1]$ but all $\theta_{i}($.$) and b_{i}($.$) map into two different payoff values and three different$ belief values, the strong injectivity property of $b_{i}: T_{i} \rightarrow \mathcal{M}\left(T_{-i}\right)$ is not necessary to extract surplus from at most 6 different pairs of payoffs and beliefs.

11 With arbitrary abstract type spaces, one may have reservations about participation fee schedules $z_{n}^{i}$ that condition on the other agents' abstract types. These types may not be observable and verifiable. To take account of this objection, one can have the participation fee schedules condition on verifiable messages $m_{j}\left(t_{j}\right)$ that may but need not reflect the other agents' payoff parameters and/or beliefs. Expected payments then depend on $b_{i}\left(t_{i}\right)$ through the induced distribution $b_{i}\left(t_{i}\right) \circ m_{-i}^{-1}(\cdot)$ of message vectors, where, for $t_{-i} \in T_{-i}$, $m_{-i}\left(t_{. i}\right)=\left(m_{j}\left(t_{i}\right)\right)_{j \neq i}$. Our analysis goes through unchanged, except that the assumption below about type spaces having more than finitely many elements must be replaced by an assumption that the ranges of the mappings $m_{i}, i=1, \ldots, I$, have more than finitely many elements.
} 
THEOREM 2.2 Assume that $T_{i}$ is compact. Assume also that the map (11) maps $T_{i}$ continuously into the space $\Theta_{i} \times \mathcal{M}\left(T_{-i}\right)$ where $\mathcal{M}\left(T_{-i}\right)$ is endowed with a topology that is at least as fine as the topology of weak convergence of probability measures. Then the pair $\left(\theta_{i}(\cdot), b_{i}(\cdot)\right)$ has the FSE* property if and only if, for every $\bar{t}_{i} \in T_{i}$ and every probability measure $\mu$ on $T_{i}$,

$$
\varphi_{b_{i}}(\mu)=\varphi_{b_{i}}\left(\delta_{\bar{t}_{i}}\right) \text { implies } \mu \circ\left(\theta_{i}(\cdot), b_{i}(\cdot)\right)^{-1}=\delta_{\left(\theta_{i}\left(\bar{t}_{i}\right), b_{i}\left(\bar{t}_{i}\right)\right)},
$$

where $\delta_{\left(\theta_{i}\left(\bar{t}_{i}\right), b_{i}\left(\bar{t}_{i}\right)\right)}$ is the degenerate measure that assigns all mass to the the singleton $\left\{\left(\theta_{i}\left(\bar{t}_{i}\right), b_{i}\left(\bar{t}_{i}\right)\right)\right\}$ and $\varphi_{b_{i}}\left(\mu_{i}\right)$ is defined as in Theorem 2.1.

Proof. If $T_{i}$ is compact, the range $R\left(\theta_{i}(\cdot), b_{i}(\cdot)\right)$ of the continuous function (11), a subset of the metric space $\Theta_{i} \times \mathcal{M}\left(T_{-i}\right)$, is also compact. We may think of $R\left(\theta_{i}(\cdot), b_{i}(\cdot)\right)$ as a type space in its own right and of the projection from $R\left(\theta_{i}(\cdot), b_{i}(\cdot)\right)$ to $\mathcal{M}\left(T_{-i}\right)$ as a belief mapping. One easily verifies that the pair $\left(\theta_{i}(\cdot), b_{i}(\cdot)\right)$ has the $\mathrm{FSE}^{*}$ property if and only if the projection from $R\left(\theta_{i}(\cdot), b_{i}(\cdot)\right)$ to $\mathcal{M}\left(T_{-i}\right)$ has the FSE property as defined in Section 2.1. By Theorem 2.1, the projection from $R\left(\theta_{i}(\cdot), b_{i}(\cdot)\right)$ to $\mathcal{M}\left(T_{-i}\right)$ has the FSE property if and only if, for every pair $\left(\bar{\theta}_{i}, \bar{b}_{i}\right) \in R\left(\theta_{i}(\cdot), b_{i}(\cdot)\right)$ and every probability measure $\mu^{*}$ on $R\left(\theta_{i}(\cdot), b_{i}(\cdot)\right)$,

$$
\varphi_{\operatorname{proj}_{\mathcal{M}\left(T_{-i}\right)}}\left(\mu^{*}\right)=\varphi_{\operatorname{proj}_{\mathcal{M}\left(T_{-i}\right)}}\left(\delta_{\left(\bar{\theta}_{i}, \bar{b}_{i}\right)}\right) \text { implies } \mu^{*}=\delta_{\left(\bar{\theta}_{i}, \bar{b}_{i}\right)}
$$

We further note that, by the definition of $R\left(\theta_{i}(\cdot), b_{i}(\cdot)\right)$, a pair $\left(\bar{\theta}_{i}, \bar{b}_{i}\right)$ belongs to $R\left(\theta_{i}(\cdot), b_{i}(\cdot)\right)$ if and only if there exists $\bar{t}_{i} \in T_{i}$ such that $\left(\bar{\theta}_{i}, \bar{b}_{i}\right)=\left(\theta_{i}\left(\bar{t}_{i}\right), b_{i}\left(\bar{t}_{i}\right)\right)$, and a measure $\mu^{*}$ belongs to $\mathcal{M}\left(R\left(\theta_{i}(\cdot), b_{i}(\cdot)\right)\right)$ if and only if there exists $\mu \in \mathcal{M}\left(T_{i}\right)$ such that $\mu^{*}=\mu \circ$ $\left(\theta_{i}(\cdot), b_{i}(\cdot)\right)^{-1}$. Moreover, $\left(\bar{\theta}_{i}, \bar{b}_{i}\right)=\left(\theta_{i}\left(\bar{t}_{i}\right), b_{i}\left(\bar{t}_{i}\right)\right)$ and $\mu^{*}=\mu \circ\left(\theta_{i}(\cdot), b_{i}(\cdot)\right)^{-1}$ imply

$$
\varphi_{\operatorname{proj}_{\mathcal{M}\left(T_{-i}\right)}}\left(\delta_{\left(\bar{\theta}_{i}, \bar{b}_{i}\right)}\right)=\varphi_{b_{i}}\left(\delta_{\bar{t}_{i}}\right) \text { and } \varphi_{\operatorname{proj}_{\mathcal{M}\left(T_{-i}\right)}}\left(\mu^{*}\right)=\varphi_{b_{i}}(\mu)
$$

and, hence,

$$
\varphi_{\operatorname{proj}_{\mathcal{M}\left(T_{-i}\right)}}\left(\mu^{*}\right)=\varphi_{\operatorname{proj}_{\mathcal{M}\left(T_{-i}\right)}}\left(\delta_{\left(\bar{\theta}_{i}, \bar{b}_{i}\right)}\right) \text { if and only if } \varphi_{b_{i}}(\mu)=\varphi_{b_{i}}\left(\delta_{\bar{t}_{i}}\right) .
$$

Thus, (12) is equivalent to condition $(* *)$.

We refer to condition ( $\left.{ }^{* *}\right)$ as the generalized McAfee-Reny condition. This condition is obviously weaker than the McAfee-Reny condition itself. The following corollary provides a decomposition of condition $\left(^{* *}\right)$ that makes the underlying structure more transparent. 
COROLlaRY 2.3 Under the assumptions of Theorem 2.2, the function $\left(\theta_{i}(\cdot), b_{i}(\cdot)\right)$ from $T_{i}$ to $\Theta_{i} \times \mathcal{M}\left(T_{-i}\right)$ has the FSE* property if and only if the following two conditions hold:

(a) for every $\bar{t}_{i} \in T_{i}$ and every probability measure $\mu$ on $T_{i}$,

$$
\varphi_{b_{i}}(\mu)=\varphi_{b_{i}}\left(\delta_{\bar{t}_{i}}\right) \text { implies } \varphi_{b_{i}}(\mu) \circ b_{i}^{-1}=\delta_{b_{i}\left(\bar{t}_{i}\right)}
$$

i.e. all types $t_{i}$ in the support of $\mu$ have the same beliefs $b_{i}\left(t_{i}\right)=b_{i}\left(\bar{t}_{i}\right)$;

(b) for any two types $t_{i}$ and $t_{i}^{\prime}$ in $T_{i}$

$$
\theta_{i}\left(t_{i}\right) \neq \theta_{i}\left(t_{i}^{\prime}\right) \text { implies } b_{i}\left(t_{i}\right) \neq b_{i}\left(t_{i}^{\prime}\right) \text {. }
$$

Proof. Suppose that the pair $\left(\theta_{i}(\cdot), b_{i}(\cdot)\right)$ satisfies (a) and (b). If $\bar{t}_{i} \in T_{i}$ and $\mu \in \mathcal{M}\left(T_{i}\right)$ are such that $\varphi_{b_{i}}(\mu)=\varphi_{b_{i}}\left(\delta_{\bar{t}_{i}}\right)$, then (a) implies that all types in in the support of $\mu$ have beliefs equal to $b_{i}\left(\bar{t}_{i}\right)$. By (b), it follows that all types in the support of $\mu$ have payoff parameters equal to $\theta_{i}\left(\bar{t}_{i}\right)$. The measure $\mu$ is thus concentrated on the set of types with payoff-belief pairs equal to $\left(\theta_{i}\left(\bar{t}_{i}\right), b_{i}\left(\bar{t}_{i}\right)\right)$. Thus, if (a) and (b) hold, the generalized McAfee-Reny condition is satisfied.

Conversely, if the generalized McAfee-Reny condition holds, (a) follows immediately. To see that (b) must also hold, suppose that there exist two types $t_{i}$ and $t_{i}^{\prime}$ in $T_{i}$ such that $\theta_{i}\left(t_{i}\right) \neq \theta_{i}\left(t_{i}^{\prime}\right)$ and $b_{i}\left(t_{i}\right)=b_{i}\left(t_{i}^{\prime}\right)$. Let $\mu=\frac{1}{2} \delta_{t_{i}}+\frac{1}{2} \delta_{t_{i}^{\prime}}$. Then $\varphi_{b_{i}}(\mu)=\varphi_{b_{i}}\left(\delta_{t_{i}}\right)$ but $\mu \circ\left(\theta_{i}(\cdot), b_{i}(\cdot)\right)^{-1} \neq \delta_{\left(\theta_{i}\left(\bar{t}_{i}\right), b_{i}\left(\bar{t}_{i}\right)\right)}$, i.e. the generalized McAfee-Reny condition does not hold.

Condition (b) corresponds to what Neeman (2004) and Heifetz and Neeman (2006) call the BDP property - "beliefs determine preferences": If one knows an agent's beliefs, then one can infer the agent's payoff parameters. The necessity of this property for full surplus extraction was originally established by Neeman (2004) and Heifetz and Neeman (2006).

Condition (a) can be interpreted as a screening condition, like the McAfee-Reny condition but somewhat weaker. Whereas the McAfee-Reny condition requires that the belief $b_{i}\left(\bar{t}_{i}\right)$ of any type $\bar{t}_{i} \in T_{i}$ must be distinguishable from the beliefs that are associated with any extended type, the generalized McAfee-Reny condition only requires that $b_{i}\left(\bar{t}_{i}\right)$ must be distinguishable from any extended type that assigns positive probability to payoff parameters different from $\theta_{i}\left(\bar{t}_{i}\right)$. 


\subsection{Genericity of the FSE and FSE* Properties}

We study the genericity of full surplus extraction in terms of the set of belief functions having the FSE property. Because a pair $\left(\theta_{i}(\cdot), b_{i}(\cdot)\right)$ of functions mapping abstract types into payoff parameters and beliefs has the FSE ${ }^{*}$ property whenever $b_{i}(\cdot)$ has the FSE property, any genericity result for belief functions $b_{i}$ that have the FSE property is easily translated into a genericity result for pairs $\left(\theta_{i}(\cdot), b_{i}(\cdot)\right)$ that have the $\mathrm{FSE}^{*}$ property.

We consider belief functions $b_{i}: T_{i} \rightarrow \mathcal{M}\left(T_{-i}\right)$ that are continuous. The meaning of this assumption depends on the topology that is imposed on $\mathcal{M}\left(T_{-i}\right)$, the space of probability measures on $T_{-i}:=\prod_{j \neq i} T_{j}$ with the Borel $\sigma$-algebra $\mathcal{B}\left(T_{-i}\right)=\prod_{j \neq i} \mathcal{B}\left(T_{j}\right)$. In principle, the topology should be specified so as to properly reflect the continuity properties of the functions and correspondences that one is interested in. Given the restriction to continuous payoff functions $\pi_{i}$ and given the use of continuous participation fee schedules $z_{n}^{i}$, a natural candidate for a topology on $\mathcal{M}\left(T_{-i}\right)$ is the topology of weak convergence of probability measures, under which the mappings from measures into integrals of bounded continuous functions are continuous. ${ }^{12}$ With this topology, participants' objective functions can usually be taken to be continuous and behaviour correspondences upper hemicontinuous in their beliefs.

Use of the topology of weak convergence has however been criticized because this topology is too coarse to provide for what Dekel, Fudenberg, and Morris (2006) refer to as the lower semicontinuity property, i.e. the property that the minimal $\varepsilon \geq 0$ for which strategies are interim $\varepsilon$-rationalizable should depend continuously on their types. ${ }^{13}$ This

\footnotetext{
${ }^{12}$ As is well known, if $T_{-i}$ is a separable metric space, $\mathcal{M}\left(T_{-i}\right)$ can be identified with the space of continuous linear functionals on the space $\mathcal{C}\left(T_{-i}\right)$ of bounded continuous real-valued functions on $T_{-i}$, i.e. the dual of $\mathcal{C}\left(T_{-i}\right)$, and the topology of weak convergence coincides with the weak topology. If $T_{-i}$ is not separable, the dual of $\mathcal{C}\left(T_{-i}\right)$ corresponds to the space $r b a\left(T_{-i}\right)$ of regular (finitely) additive set functions on $\left(T_{-i}, \mathcal{B}\left(T_{-i}\right)\right)$, which is larger than $\mathcal{M}\left(T_{-i}\right)$. In this case, the topology of weak convergence coincides with the subspace topology that is induced by the weak* topology on $r b a\left(T_{-i}\right)$. See, e.g., Parthasarathy (1967), p. 35.

${ }^{13}$ See Dekel, Fudenberg, and Morris (2006), Chen, DiTillio, Faingold, and Xiong (2010). Their criticism of the weak ${ }^{*}$ topology is formulated in the context of the universal type space, but applies in abstract type space as well. As an example, consider the following version of Rubinstein (1989) e-mail game. Let $I=2$, and set $T_{1}=T_{2}=\left\{0, \frac{1}{2}, \frac{2}{3}, \ldots, 1\right\}$. Suppose that each agent has action set $\{0,1\}$ and that the payoffs are given as $(0,0)$ if both agents choose the action $a_{i}=0(1,1)$ if $t_{1}>0$ and both agents choose the action $a_{i}=1$, and $(-x,-x)$ in all other cases, i.e. if $t_{1}=0$ or if $t_{1}>0$ and $a_{i} \neq a_{-i}$.

Specify a belief function $b_{1}$ for agent 1 so that, for some $\alpha \in(0,1)$ and $n=1,2, \ldots, b_{1}\left(\frac{n}{n+1}\right)=\alpha \delta_{(n-1) / n}+$ $(1-\alpha) \delta_{n /(n+1)}$ and $b_{1}(1)=\delta_{1}$, where for any $t \in[0,1], \delta_{t}$ is the degenerate measure that assigns all proba-
} 
criticism would call for the use of a finer topology.

We do not actually specify a particular topology on $\mathcal{M}\left(T_{-i}\right)$ but assume that the topology on $\mathcal{M}\left(T_{-i}\right)$ is metrizable by a metric that is a convex function on $\mathcal{M}\left(T_{-i}\right) \times \mathcal{M}\left(T_{-i}\right)$. This assumption includes the topology of weak convergence of probability measures, which is metrizable by the metric

$$
\rho^{B L}(\beta, \hat{\beta})=\sup _{f}\left|\int_{T_{-i}} f\left(t_{-i}\right) \beta\left(d t_{-i}\right)-\int_{T_{-i}} f\left(t_{-i}\right) \hat{\beta}\left(d t_{-i}\right)\right|
$$

where the supremum is taken over the set of bounded Lipschitz continuous functions $f: T_{-i} \rightarrow \mathbb{R}$ for which

$$
\sup _{t_{-i} \in T_{-i}}\left|f\left(t_{-i}\right)\right|+\sup _{\substack{t_{-i} \in T_{-i} \\ \hat{t}_{-i} \in T_{-i}}} \frac{\left|f\left(t_{-i}\right)-f\left(\hat{t}_{-i}\right)\right|}{d\left(t_{-i}, \hat{t}_{-i}\right)} \leq 1,
$$

where $d$ is the metric on $T_{-i}{ }^{14}$ The function $\rho^{B L}$ is obviously convex.

Metrizability by a convex metric is also satisfied by any topology that can be induced by a metric of the form

$$
\rho(\beta, \hat{\beta})=\|\beta-\hat{\beta}\|,
$$

where $\|\cdot\|$ is a norm on the space of signed measures on $T_{-i}$. An example is the metric

$$
\rho^{T V}(\beta, \hat{\beta})=\sup _{B \in \mathcal{B}\left(T_{-i}\right)}|\beta(B)-\hat{\beta}(B)|
$$

which identifies the distance between two measures $\beta$ and $\hat{\beta}$ with the total variation of the signed measure $\beta-\hat{\beta}$. The topology that induced by the total-variation metric is immune

bility mass to the singleton $\{t\}$. Similarly, specify a belief function $b_{2}$ for agent 2 so that, for some $\beta \in(0,1)$ and $n=1,2, \ldots, b_{2}\left(\frac{n}{n+1}\right)=\beta \delta_{n /(n+1)}+(1-\beta) \delta_{(n+1) /(n+2)}$ and $b_{2}(1)=\delta_{1}$.

If the measures $b_{1}\left(t_{1}\right), t_{1} \in T_{1}$, are absolutely continuous with respect to some fixed measure $\lambda$ on $T_{2}$, then, for $n=1,2, \ldots$, the density of $b_{1}\left(\frac{n}{n+1}\right)$ with respect to $\lambda$ satisfies $f_{b_{1}}\left(\frac{n}{n+1} \mid \frac{n}{n+1}\right)=(1-\alpha) / \lambda\left(\left\{\frac{n}{n+t}\right\}\right)$. Because $\lambda$ is a probability measure, it must be the case that $\lambda\left(\left\{\frac{n}{n+t}\right\}\right)$ goes to zero as $n$ goes out of bounds. Hence $f_{b_{1}}\left(\frac{n}{n+1} \mid \frac{n}{n+1}\right)$ fails to converge to $f_{b_{1}}(1 \mid 1)=1 / \lambda(\{1\})$ as $n$ goes out of bounds and $\frac{n}{n+t}$ converges to one.

One easily verifies that, if $x$ is sufficiently large, then for each agent $i$, the action $a_{i}=1$ is interim rationalizable if $t_{i}=1$ but not if $t_{i}<1$. Indeed, if $\varepsilon>0$ is sufficiently small, then $a_{i}=1$ is not even $\varepsilon$-rationalizable if $t_{i}<1$. We return to the issue in the context of the universal type space in Section 3.

${ }^{14}$ See Dudley (2002), p. 395. 
to the criticism of Dekel, Fudenberg, and Morris (2006). ${ }^{15}$ The proof that the functions $\rho^{w}$ and $\rho^{T V}$ are convex is straightforward and is left to the reader.

Given the topology on $\mathcal{M}\left(T_{-i}\right)$, we write $\mathcal{C}\left(T_{i}, \mathcal{M}\left(T_{-i}\right)\right)$ for the space of continuous belief functions from $T_{i}$ to $\mathcal{M}\left(T_{-i}\right)$, and we endow this space with the topology of uniform convergence. We also write $\mathcal{E}^{*}\left(T_{i}, \mathcal{M}\left(T_{-i}\right)\right)$ for the set of those belief functions in $\mathcal{C}\left(T_{i}, \mathcal{M}\left(T_{-i}\right)\right)$ that satisfy the McAfee-Reny condition $\left(^{*}\right)$ for full surplus extraction. Throughout the remainder of the paper, we impose the following assumption.

Assumption Each of the type spaces $T_{i}$ has more than finitely many elements.

If the type spaces are finite sets and $T_{-i}$ has at least as many elements as $T_{i}$, the analysis of Crémer and McLean (1988) implies that $\mathcal{E}^{*}\left(T_{i}, \mathcal{M}\left(T_{-i}\right)\right)$ is actually an open and dense subset of $\mathcal{C}\left(T_{i}, \mathcal{M}\left(T_{-i}\right)\right) .{ }^{16}$ With infinite type spaces, we only obtain residualness and denseness.

THEOREM 2.4 Assume that the metric space $T_{i}$ is compact. Assume also that the topology on $\mathcal{M}\left(T_{-i}\right)$ is at least as fine as the topology of weak convergence and is induced by a metric that is a convex function on $\mathcal{M}\left(T_{-i}\right) \times \mathcal{M}\left(T_{-i}\right)$. Then the set $\mathcal{E}^{*}\left(T_{i}, \mathcal{M}\left(T_{-i}\right)\right)$ of continuous functions from $T_{i}$ to $\mathcal{M}\left(T_{-i}\right)$ that have the FSE property is a residual subset of the space $\mathcal{C}\left(T_{i}, \mathcal{M}\left(T_{-i}\right)\right)$, i.e., $\mathcal{E}^{*}\left(T_{i}, \mathcal{M}\left(T_{-i}\right)\right)$ contains a countable intersection of open and dense subsets of $\mathcal{C}\left(T_{i}, \mathcal{M}\left(T_{-i}\right)\right)$. If the metric space $\mathcal{M}\left(T_{-i}\right)$ is complete, $\mathcal{E}^{*}\left(T_{i}, \mathcal{M}\left(T_{-i}\right)\right)$ is itself dense in $\mathcal{C}\left(T_{i}, \mathcal{M}\left(T_{-i}\right)\right)$.

Theorem 2.4 is the major mathematical contribution of this paper. Its proof is given at the end of our discussion of abstract type spaces, in Section 2.5 below.

To complete the discussion here, we note that, by the arguments given above, the set $\mathcal{C}\left(T_{i}, \Theta_{i}\right) \times \mathcal{E}^{*}\left(T_{i}, \mathcal{M}\left(T_{-i}\right)\right)$ is contained in a subset of the set $\mathcal{E}^{* *}\left(T_{i}, \Theta_{i} \times \mathcal{M}\left(T_{-i}\right)\right)$ of pairs $\left(\theta_{i}(\cdot), b_{i}(\cdot)\right)$ that have the $\mathrm{FSE}^{*}$ property. Thus Theorem 2.4 immediately yields:

\footnotetext{
${ }^{15}$ Engl (1995) shows that, if beliefs have the topology of set-wise convergence, then the Nash equilibrium correspondence has the required lower semi-continuity property. Engl's arguments are easily extended to the correspondence of interim $\varepsilon$-rationalizable actions. Since the topology of setwise convergence is coarser than the topology induced by the total-variation metric, the lower semi-continuity property also holds if beliefs are endowed with the latter topology.

${ }^{16}$ For the case of finite type sets, Crémer and McLean (1988) also showed that full surplus extraction through a dominant-strategy mechanism can be achieved if and only if the matrix of posterior beliefs of all types of each agent has the rank $n_{i}$ where $n_{i}$ is the cardinality of the type space of agent $i$.
} 
COROLlaRY 2.5 Under the assumptions of Theorem 2.4, the set $\mathcal{E}^{* *}\left(T_{i}, \Theta_{i} \times \mathcal{M}\left(T_{-i}\right)\right)$ of continuous functions from $T_{i}$ to $\Theta_{i} \times \mathcal{M}\left(T_{-i}\right)$ that have the FSE* property is a residual subset of the space $\mathcal{C}\left(T_{i}, \Theta_{i} \times \mathcal{M}\left(T_{-i}\right)\right)$, i.e. $\mathcal{E}^{* *}\left(T_{i}, \Theta_{i} \times \mathcal{M}\left(T_{-i}\right)\right)$ contains a countable intersection of open and dense subsets of $\mathcal{C}\left(T_{i}, \Theta_{i} \times \mathcal{M}\left(T_{-i}\right)\right)$. If the metric space $\Theta_{i} \times \mathcal{M}\left(T_{-i}\right)$ is complete, $\mathcal{E}^{* *}\left(T_{i}, \Theta_{i} \times \mathcal{M}\left(T_{-i}\right)\right)$ is itself dense in $\mathcal{C}\left(T_{i}, \Theta_{i} \times \mathcal{M}\left(T_{-i}\right)\right)$.

\subsection{Genericity of Common Priors with the FSE Property}

Whereas the analysis so far has focused on a single agent, we now consider the scope for surplus extraction from all participants together. We restrict our analysis to the case where the belief functions $b_{i}, i=1, \ldots, I$, can be interpreted as regular conditional dis-

tributions that are derived from a common prior on the space $T:=\prod_{i=1}^{I} T_{i}$ of vectors of all agents' types. As before, for any $i$, we allow $\mathcal{M}\left(T_{-i}\right)$ to have any topology that is at least as fine as the weak ${ }^{*}$ topology and is induced by a metric that is a convex function on $\mathcal{M}\left(T_{-i}\right) \times \mathcal{M}\left(T_{-i}\right)$. The belief function $b_{i}$ is again treated as an element of the space $\mathcal{C}\left(T_{i}, \mathcal{M}\left(T_{-i}\right)\right)$ of continuous functions from $T_{i}$ to $\mathcal{M}\left(T_{-i}\right)$. We consider priors that have marginal distributions with full supports, $T_{1}, \ldots, T_{I}$, and that admit continuous regular conditional distributions for $t_{-i}$ given $t_{i}$, for all $i$. The set of such priors is denoted as $\mathcal{M}_{f}^{c}(T)$.

REMARK 2.6 For any $\mu \in \mathcal{M}_{f}^{c}(T)$ and any $i$, there exist a unique $\mu_{i}(\mu) \in \mathcal{M}\left(T_{i}\right)$ and a unique $b_{i}(\mu) \in \mathcal{C}\left(T_{i}, \mathcal{M}\left(T_{-i}\right)\right)$ such that, under the prior $\mu, \mu_{i}(\mu)$ is the marginal distribution on $T_{i}$ and $b_{i}(\mu)$ is a regular conditional distribution for $t_{-i}$ given $t_{i}$.

Proof. Fix $\mu \in \mathcal{M}_{f}^{c}(T)$. Existence and uniqueness of the marginal distributions $\mu_{i}(\mu)$, $i=1, \ldots, I$ are standard. The definition of $\mathcal{M}_{f}^{c}(T)$ implies that, for any $i$, there also exists a function $b_{i}(\mu) \in \mathcal{C}\left(T_{i}, \mathcal{M}\left(T_{-i}\right)\right)$ that is a regular conditional distribution for $t_{-i}$ given $t_{i}$. Consider any other function $\hat{b}_{i}$ in $\mathcal{C}\left(T_{i}, \mathcal{M}\left(T_{-i}\right)\right)$. If $\hat{b}_{i} \neq b_{i}(\mu)$, there exists $t_{i} \in T_{i}$ such that $\hat{b}_{i}\left(t_{i}\right) \neq b_{i}\left(t_{i}, \mu\right)$. Because $\hat{b}_{i}$ and $b_{i}(\mu)$ are both continuous functions, it follows that $\hat{b}_{i}\left(t_{i}^{\prime}\right) \neq b_{i}\left(t_{i}^{\prime}, \mu\right)$ for all $t_{i}^{\prime}$ in an open neighbourhood of $t_{i}$. Because $\mu$ has full support, $t_{i}$ belongs to the support of $\mu_{i}(\mu)$, and we must have $\mu\left(\left\{t_{i}^{\prime} \in T_{i} \mid \hat{b}_{i}\left(t_{i}^{\prime}\right) \neq b_{i}\left(t_{i}^{\prime}, \mu\right)\right\}\right)>0$. Since regular conditional distributions induced by a given prior coincide almost surely, it follows that $\hat{b}_{i}$ cannot be a regular conditional distribution for $t_{-i}$ given $t_{i}$ under the prior 
$\mu$, i.e., $b_{i}(\mu)$ is the only continuous function that is a regular conditional distribution for $t_{-i}$ given $t_{i}$ under the measure $\mu$.

For simplicity, we focus on the FSE property rather than the FSE* property. Given the mappings $\mu \rightarrow b_{i}(\mu)$ from $\mathcal{M}_{f}^{c}(T)$ to $\mathcal{C}\left(T_{i}, \mathcal{M}\left(T_{-i}\right)\right), i=1, \ldots, I$, we say that a prior $\mu$ admits full surplus extraction if and only if each of the belief functions $b_{i}(\mu), i=1, \ldots, I$, has the FSE property. We refer to such priors as FSE priors and denote the set of FSE priors on $T$ as $\mathcal{F}(T)$. The following theorem establishes the genericity of FSE priors in $\mathcal{M}_{f}^{c}(T)$.

THEOREM 2.7 Assume for each $i$, that $T_{i}$ is a compact metric space and that the topology on $\mathcal{M}\left(T_{-i}\right)$ is at least as fine as the topology of weak convergence and is induced by a metric that is a convex function on $\mathcal{M}\left(T_{-i}\right) \times \mathcal{M}\left(T_{-i}\right)$. Let $\mathcal{M}_{f}^{c}(T)$ be endowed with the coarsest topology under which each of the mappings $\mu \rightarrow \mu_{i}(\mu), \mu \rightarrow b_{i}(\mu), i=1, \ldots, I$, is continuous. Then the set $\mathcal{F}(T)$ of FSE priors is a residual and dense subset of $\mathcal{M}_{f}^{c}(T)$, i.e., $\mathcal{F}(T)$ contains a countable intersection of open and dense subsets of $\mathcal{M}_{f}^{c}(T)$. If the spaces $\mathcal{M}\left(T_{-i}\right)$ are complete, the set $\mathcal{F}(T)$ is itself dense.

Proof. For $i=1, \ldots, I$, let $\mathcal{F}_{i}(T) \subset \mathcal{M}_{f}^{c}(T)$ be the set of priors $\mu$ for which the belief function $b_{i}(\mu)$ belongs to the set $\mathcal{E}^{*}\left(T_{i}, \mathcal{M}\left(T_{-i}\right)\right)$ of functions in $\mathcal{C}\left(T_{i}, \mathcal{M}\left(T_{-i}\right)\right.$ that satisfy the McAfee-Reny condition for full surplus extraction. Clearly,

$$
\mathcal{F}(T)=\bigcap_{i=1}^{I} \mathcal{F}_{i}(T) .
$$

To prove that $\mathcal{F}(T)$ is a residual subset of $\mathcal{M}_{f}^{c}(T)$, it is therefore enough to show that each of the sets $\mathcal{F}_{i}(T), i=1, \ldots, I$, contains a countable intersection of open and dense subsets of $\mathcal{M}_{f}^{c}(T)$.

We claim that, with the specified topology on $\mathcal{M}_{f}^{c}(T)$, for any $i$, the map $\mu \rightarrow b_{i}(\mu)$ is open as well as continuous. To see this, it suffices to note that this map is the composition of the map $\mu \rightarrow\left\{\mu_{j}(\mu), b_{j}(\mu)\right\}_{j=1}^{I}$ from $\mathcal{M}_{f}^{c}(T)$ to $\prod_{j=1}^{I}\left[\mathcal{M}\left(T_{i}\right) \times \mathcal{C}\left(T_{j}, \mathcal{M}\left(T_{-j}\right)\right)\right]$ and the projection from $\prod_{j=1}^{I}\left[\mathcal{M}\left(T_{i}\right) \times \mathcal{C}\left(T_{j}, \mathcal{M}\left(T_{-j}\right)\right)\right]$ to $\mathcal{C}\left(T_{i}, \mathcal{M}\left(T_{-i}\right)\right)$. The topology on $\mathcal{M}_{f}^{c}(T)$ is specified so that the open subsets of $\mathcal{M}_{f}^{c}(T)$ are exactly those sets $V$ for which the image sets $\left\{\left\{\mu_{j}(\mu), b_{j}(\mu)\right\}_{j=1}^{I} \mid \mu \in V\right\}$ are open in $\prod_{j=1}^{I}\left[\mathcal{M}\left(T_{i}\right) \times \mathcal{C}\left(T_{j}, \mathcal{M}\left(T_{-j}\right)\right)\right]$. Therefore the map $\mu \rightarrow\left\{\mu_{j}(\mu), b_{j}(\mu)\right\}_{j=1}^{I}$ is open as well as continuous. Since the projection is also open and continuous, it follows that, for any $i$, the map $\mu \rightarrow b_{i}(\mu)$ is open and continuous. 
For any $i$, Theorem 2.4 implies that the set $\mathcal{E}^{*}\left(T_{i}, \mathcal{M}^{\lambda}\left(T_{-i}\right)\right)$ contains a sequence $\left\{W_{k}^{i}\right\}_{k=1}^{\infty}$ of open and dense subsets of $\mathcal{C}\left(T_{i}, \mathcal{M}\left(T_{-i}\right)\right.$. For any $i$ and $k$, define

$$
V_{k}^{i}:=\left\{\mu \in \mathcal{M}_{f}^{c}(T) \mid b_{i}(\mu) \in W_{k}^{i}\right\}
$$

By the definition of $\mathcal{F}_{i}(T), V_{k}^{i} \subset \mathcal{F}_{i}(T)$ for all $k$. Hence, $\cap_{k=1}^{\infty} V_{k}^{i} \subset \mathcal{F}_{i}(T)$. Because $W_{k}^{i}$ is open and the function $\mu \rightarrow b_{i}(\mu)$ is continuous, $V_{k}^{i}$ is also open for each $k$. Because the function $v \rightarrow b_{i}(\cdot, v)$ is open, the set

$$
\left\{b_{i}(\mu) \in \mathcal{C}\left(T_{i}, \mathcal{M}\left(T_{-i}\right) \mid \mu \in V^{*}\right\}\right.
$$

is open whenever $V^{*}$ is an open subset of $\mathcal{M}_{f}^{c}(T)$. Since $W_{k}^{i}$ is dense, it follows that the intersection

$$
W_{k}^{i} \cap\left\{b_{i}(\mu) \in \mathcal{C}\left(T_{i}, \mathcal{M}\left(T_{-i}\right) \mid \mu \in V^{*}\right\}\right.
$$

is non-empty, and therefore also the intersection $V_{k}^{i} \cap V^{*}$ is non-empty whenever $V^{*}$ is open. Thus $V_{k}^{i}$ is dense as well as open in $\mathcal{M}_{f}^{c}(T)$. It follows that $\mathcal{F}_{i}(T)$ contains a countable intersection of open and dense subsets of $\mathcal{M}_{f}^{c}(T)$ By (15) therefore $\mathcal{F}(T)$ is a residual subset of $\mathcal{M}_{f}^{c}(T)$.

For any $i$, if $\mathcal{M}\left(T_{-i}\right)$ is complete, then, by Theorem $2.4, \mathcal{E}^{*}\left(T_{i}, \mathcal{M}\left(T_{-i}\right)\right)$ is dense in $\mathcal{C}\left(T_{i}, \mathcal{M}\left(T_{-i}\right)\right)$. Trivially also, $\mathcal{M}\left(T_{i}\right) \times \mathcal{E}^{*}\left(T_{j}, \mathcal{M}\left(T_{-j}\right)\right)$ is dense in $\mathcal{M}\left(T_{i}\right) \times \mathcal{C}\left(T_{j}, \mathcal{M}\left(T_{-j}\right)\right)$. Because the map $\mu \rightarrow\left(\mu_{i}(\mu), b_{i}(\mu)\right.$ from $\mathcal{M}_{f}^{c}(T)$ to $\mathcal{M}\left(T_{i}\right) \times \mathcal{C}\left(T_{j}, \mathcal{M}\left(T_{-j}\right)\right)$ is open and continuous, it follows that $\mathcal{F}_{i}(T)$ is dense in $\mathcal{M}_{f}^{c}(T)$. Because a finite intersection of dense sets is itself dense, it follows that, if all the spaces $\mathcal{M}\left(T_{-i}\right)$ are complete, then $\mathcal{F}(T)$ is also dense in $\mathcal{M}_{f}^{c}(T)$.

In Theorem 2.7, the topology on $\mathcal{M}_{f}^{c}(T)$ depends on the topologies that are imposed on $\mathcal{M}\left(T_{-i}\right), i=1, \ldots, I$. As in Theorem 2.4 , these topologies are not actually specified; only a requirement of metrizability by a convex metric is imposed. As mentioned, this convexity requirement is satisfied, e.g., be the weak* topology and by the total-variation topology.

One easily verifies that, if $\mathcal{M}\left(T_{-i}\right)$ is endowed with the weak* topology, then the topology on $\mathcal{M}_{f}^{c}(T)$ that is defined by the continuity requirement in Theorem 2.7 is also the weak* topology. However, if $\mathcal{M}\left(T_{-i}\right)$ is endowed with the total-variation topology, the topology on $\mathcal{M}_{f}^{c}(T)$ that is defined by the continuity requirement in Theorem 2.7 is obviously finer than the weak' topology. Because the ranges $\mathcal{M}\left(T_{i}\right)$ of the mappings $\mu \rightarrow \mu_{i}(\mu), i=1, \ldots, I$, have the weak* topology, rather than the total variation topology, 
the topology on $\mathcal{M}_{f}^{c}(T)$ that is defined by the continuity requirement in Theorem 2.7 is also coarser than the total-variation topology on $\mathcal{M}_{f}^{c}(T)$ itself. ${ }^{17}$

Given that the FSE property for belief functions is stronger than the FSE* property for pairs of payoff and belief functions, Theorem 2.7 can be used to obtain a genericity result for the $\mathrm{FSE}^{*}$ property in abstract type spaces with common priors. The argument is similar to the one that was used to derive Corollary 2.5 from Theorem 2.4. The details are left to the reader.

\subsection{Proof of Theorem 2.4}

The proof of Theorem 2.4 involves ideas from the topological theory of embeddings. The classical embedding theorem for continuous functions asserts that, if $X$ and $Y$ are finitedimensional compact metric spaces and if the dimension of $Y$ is strictly greater than twice the dimension of $X$, then the set of embeddings, i.e. of continuous injective functions, is a residual subset of the set of continuous functions from $X$ to $Y$ when the space of these functions has the topology of uniform convergence. ${ }^{18}$

The McAfee-Reny condition is stronger than injectivity: If the measure $\mu_{i}$ in condition $\left(^{*}\right)$ is itself degenerate, i.e., if $\mu_{i}=\delta_{t_{i}}$ for some $t_{i} \in T_{i}$, then condition $\left(^{*}\right)$ specializes to the requirement that

$$
\varphi_{b_{i}}\left(\delta_{t_{i}}\right)=\varphi_{b_{i}}\left(\delta_{\bar{t}_{i}}\right) \text { implies } \delta_{t_{i}}=\delta_{\bar{t}_{i}}
$$

which implies that the mapping $b_{i}$ is injective. The set $\mathcal{E}^{*}\left(T_{i}, \mathcal{M}\left(T_{-i}\right)\right)$ is thus a subset of the set $\mathcal{E}\left(T_{i}, \mathcal{M}\left(T_{-i}\right)\right)$ of continuous injective functions from $T_{i}$ to $\mathcal{M}\left(T_{-i}\right)$. Theorem 2.4 implies that $\mathcal{E}\left(T_{i}, \mathcal{M}\left(T_{-i}\right)\right)$ is a residual subset of $\mathcal{C}\left(T_{i}, \mathcal{M}\left(T_{-i}\right)\right)$.

Because the McAfee-Reny condition is stronger than injectivity, we cannot use the embedding theorem itself but need a new argument. The argument involves the same ideas as the proof of the classical embedding theorem, but makes essential use of the fact that the space $\mathcal{M}\left(T_{-i}\right)$ is infinite-dimensional. Even if the domain $T_{i}$ of the belief functions

\footnotetext{
${ }^{17}$ To see this point, consider the following example, which is adapted from the Supplementary Material to Chen and Xiong (2013). Let $I=2, T_{1}=[0,1] \cup\left\{\tau_{1}^{1}, \tau_{1}^{2}\right\}, T_{2}=[0,1]$. Consider the subset $\hat{\mathcal{P}}$ of measures in $\mathcal{M}_{f}^{c}(T)$ that take the form $(1-2 \alpha) \mu+\alpha \delta_{\left(\tau_{1}^{1}, \tau_{2}\right)}+\alpha \delta_{\left(\tau_{1}^{2}, \tau_{2}\right)}$ for some $\mu \in \mathcal{M}_{f}^{c}(T)$ and $\alpha \in\left(0, \frac{1}{2}\right)$, where $\tau_{2}$ is an arbitrary but fixed element of $T_{2}$. One easily verifies that, if $\mathcal{M}_{f}^{c}(T)$ has the total variation topology, then $\hat{\mathcal{P}}$ is open and dense in $\mathcal{M}_{f}^{c}(T)$. Moreover, if $\pi_{1}\left(\tau_{1}^{1}, \tau_{2}\right) \neq \pi_{1}\left(\tau_{1}^{2}, \tau_{2}\right)$, priors in $\hat{\mathcal{P}}$ do not have the FSE property.

${ }^{18}$ See, e.g., Hurewicz and Wallman (1941). In Gizatulina and Hellwig (2014), we used this theorem to show that the set of belief functions having the BDP property is residual in $\mathcal{C}\left(T_{i}, \mathcal{M}\left(T_{-i}\right)\right)$.
} 
of agent $i$ is a finite-dimensional set, the conclusion of the theorem can only be obtained if the range is infinite-dimensional. ${ }^{19}$

Let $\mathcal{M}\left(T_{i}\right)$ be the space of probability measures on $T_{i}$, endowed with the topology of weak convergence of probability measures. Because $T_{i}$ is a compact metric space, Theorem 6.5 in Parthasarathy (1967) implies that $\mathcal{M}\left(T_{i}\right)$ of is also a compact metric space. We write $d$ for the metric on $\mathcal{M}\left(T_{i}\right)$.

The metric on $\mathcal{M}\left(T_{-i}\right)$ is denoted by $\rho$. The convexity assumption in the theorem implies that, for any index set $\mathcal{L}$, any two mappings $\ell \rightarrow \beta(\ell), \ell \rightarrow \hat{\beta}(\ell)$ from $\mathcal{L}$ to $\mathcal{M}\left(T_{-i}\right)$, and any measure $\alpha$ on $\mathcal{L}$, we have

$$
\rho\left(\int_{\mathcal{L}} \beta(\ell) \alpha(d \ell), \int_{\mathcal{L}} \hat{\beta}(\ell) \alpha(d \ell)\right) \leq \int_{\mathcal{L}} \rho(\beta(\ell), \hat{\beta}(\ell)) \alpha(d \ell) .
$$

For any $\varepsilon>0$, we define $G_{\varepsilon}$ as the subset of $\mathcal{C}\left(T_{i}, \mathcal{M}\left(T_{-i}\right)\right)$ that contains all mappings $b_{i}$ with the property that, for any $t_{i} \in T_{i}$ and all $\mu \in \mathcal{M}\left(T_{i}\right)$,

$$
\varphi_{b_{i}}\left(\delta_{t_{i}}\right)=\varphi_{b_{i}}(\mu) \text { implies } d\left(\mu, \delta_{t_{i}}\right)<\varepsilon,
$$

where $d\left(\mu, \delta_{t_{i}}\right)$ is the distance between the measures $\mu$ and $\delta_{t_{i}}$

Claim 1: $\mathcal{E}^{*}\left(T_{i}, \mathcal{M}\left(T_{-i}\right)\right)=\cap_{k=1}^{\infty} G_{1 / k}$.

Proof. If $b_{i} \in G_{1 / k}$ for all $k$, then for any $t_{i} \in T_{i}$ and all $\mu \in \mathcal{M}\left(T_{i}\right), \varphi_{b_{i}}\left(\delta_{t_{i}}\right)=\varphi_{b_{i}}(\mu)$ implies $0 \leq d\left(\mu, \delta_{t_{i}}\right) \leq 1 / k$, hence $d\left(\mu, \delta_{t_{i}}\right)=0$ and $\mu=\delta_{t_{i}}$. Conversely, if $b_{i} \in \mathcal{E}^{*}\left(T_{i}, \mathcal{M}\left(T_{-i}\right)\right)$, then, by $\left(^{*}\right), \varphi_{b_{i}}\left(\delta_{t_{i}}\right)=\varphi_{b_{i}}(\mu)$ implies $d\left(\mu, \delta_{t_{i}}\right)=0<1 / k$ for all $t_{i} \in T_{i}$, all $\mu \in \mathcal{M}\left(T_{i}\right)$, and hence $b_{i} \in G_{1 / k}$ for all $k$.

Claim 2: For any $\varepsilon>0$, the set $G_{\varepsilon}$ is an open subset of $\mathcal{C}\left(T_{i}, \mathcal{M}\left(T_{-i}\right)\right)$.

Proof. Fix any $b_{i} \in G_{\varepsilon}$, and let

$$
\Gamma:=\left\{\left(t_{i}, \mu\right) \in T_{i} \times \mathcal{M}\left(T_{i}\right) \mid d\left(\mu, \delta_{t_{i}}\right) \geq \varepsilon\right\}
$$

and

$$
\eta:=\min _{\left(t_{i}, \mu\right) \in \Gamma} \rho\left(\varphi_{b_{i}}\left(\delta_{t_{i}}\right), \varphi_{b_{i}}(\mu)\right) .
$$

Because $T_{i}$ and $\mathcal{M}\left(T_{i}\right)$ are compact, the minimum in (19) is well defined, i.e., there exists $\left(t_{i}, \mu\right) \in \Gamma$ such that $d\left(\varphi_{b_{i}}\left(\delta_{t_{i}}\right), \varphi_{b_{i}}(\mu)\right)=\eta$. Since $b_{i} \in G_{\varepsilon}$ and, for $\left(t_{i}, \mu\right) \in \Gamma, d\left(\mu, \delta_{t_{i}}\right) \geq \varepsilon$, it follows that $\eta>0$.

\footnotetext{
${ }^{19}$ In Appendix $B$, we show that, for any $n$, there is a function from $[0,1]$ to $[0,1]^{n}$ that violates condition $\left(^{*}\right)$ and cannot be approximated by functions from $[0,1]$ to $[0,1]^{n}$ that satisfy condition $\left(^{*}\right)$.
} 
Now let $\bar{b}_{i}$ be any mapping in $\mathcal{C}\left(T_{i}, \mathcal{M}\left(T_{-i}\right)\right)$ such that

$$
\rho\left(\bar{b}_{i}\left(t_{i}\right), b_{i}\left(t_{i}\right)\right)<\frac{\eta}{2}
$$

for all $t_{i} \in T_{i}$, and consider the distance $\rho\left(\varphi_{\bar{b}_{i}}(\mu), \varphi_{b_{i}}(\mu)\right)$ for any $\mu \in \mathcal{M}\left(T_{i}\right)$. By (6) and (17), with $\mathcal{L}=T_{i}, \beta(\cdot)=\bar{b}_{i}(\cdot), \hat{\beta}(\cdot)=b_{i}(\cdot)$, and $\alpha=\mu$, we have

$$
\begin{aligned}
\rho\left(\varphi_{\bar{b}_{i}}(\mu), \varphi_{b_{i}}(\mu)\right) & =\rho\left(\int_{T_{i}} \bar{b}_{i}\left(t_{i}\right) \mu\left(d t_{i}\right), \int_{T_{i}} b_{i}\left(t_{i}\right) \mu\left(d t_{i}\right)\right) \\
& \leq \int_{T_{i}} \rho\left(\bar{b}_{i}\left(t_{i}\right), b_{i}\left(t_{i}\right)\right) \mu\left(d t_{i}\right) .
\end{aligned}
$$

By (20), it follows that

$$
\rho\left(\varphi_{\bar{b}_{i}}(\mu), \varphi_{b_{i}}(\mu)\right)<\frac{\eta}{2} .
$$

Thus, if $t_{i}$ and $\mu$ are such that $\varphi_{\bar{b}_{i}}\left(\delta_{t_{i}}\right)=\varphi_{\bar{b}_{i}}(\mu)$, it must be the case that $\rho\left(\varphi_{b_{i}}\left(\delta_{t_{i}}\right), \varphi_{b_{i}}(\mu)\right)<$ $\eta$. By the definition of $\eta$, it follows that $d\left(\mu, \delta_{t_{i}}\right)<\varepsilon$, which proves that $\bar{b}_{i}$ is also an element of $G_{\varepsilon}$. Thus, along with $b_{i}$, any element of the open $\frac{\eta}{2}$-ball around $b_{i}$ is an element of $G_{\varepsilon}$. The claim follows immediately.

Claim 3: For any $\varepsilon>0$, the set $G_{\varepsilon}$ is a dense subset of $\mathcal{C}\left(T_{i}, \mathcal{M}\left(T_{-i}\right)\right)$.

Proof. Let $\varepsilon>0$ be given. Fix any $b_{i} \in \mathcal{C}\left(T_{i}, \mathcal{M}\left(T_{-i}\right)\right)$ and any $\eta>0$. We will show that there exists a function $\bar{b}_{i} \in G_{\varepsilon}$ such that

$$
\rho\left(\bar{b}_{i}\left(t_{i}\right), b_{i}\left(t_{i}\right)\right)<\eta
$$

for all $t_{i} \in T_{i}$.

Relying on the fact that the continuous function $b_{i}$ is uniformly continuous on the compact set $T_{i}$, let $\zeta \in(0, \varepsilon)$ be such that, for any $t_{i}$ and $\bar{t}_{i}$ in $T_{i}, d\left(\delta_{t_{i}}, \delta_{\bar{t}_{i}}\right)<\zeta$ implies $\rho\left(b_{i}\left(t_{i}\right), b_{i}\left(t_{i}^{\prime}\right)\right)<\frac{\eta}{2}$. Because $T_{i}$ is a metric space, there exists a covering $U_{1}, U_{2}, \ldots$ of $T_{i}$ such that

$$
d\left(U_{k}\right)<\zeta
$$

and

$$
\rho\left(b_{i}\left(U_{k}\right)\right)<\frac{\eta}{2}
$$

for all $k$. where $d\left(U_{k}\right)$ and $\rho\left(b_{i}\left(U_{k}\right)\right)$ are defined as the suprema of $d\left(t_{i}, \bar{t}_{i}\right)$ and of $\rho\left(b_{i}\left(t_{i}\right), b_{i}\left(\bar{t}_{i}\right)\right)$, respectively, over $t_{i}, \bar{t}_{i}$ in $U_{k i}$.

Because $T_{i}$ is compact, the covering $U_{1}, U_{2}, \ldots$ of $T_{i}$ has a finite subcovering, which we denote as $U_{1}, U_{2}, . ., U_{K}$. Given the sets $U_{1}, U_{2}, . ., U_{K}$, we may select measures $\beta_{1}, \ldots, \beta_{K}$ in 
$\mathcal{M}\left(T_{-i}\right)$ such that, for $k=1, \ldots, K, \rho\left(\beta_{k}, b_{i}\left(\bar{t}_{i}\right)\right)<\frac{\eta}{2}$ for some $\bar{t}_{i} \in U_{k}$, and, moreover, for some continuous function $\mathbf{g}: T_{-i} \rightarrow[0,1]^{K}$, the integrals

$$
\int_{T_{-i}} \mathbf{g}\left(t_{-i}\right) d \beta_{k}\left(t_{-i}\right), k=1, \ldots, K,
$$

are linearly independent. The existence of such $\beta_{1}, \ldots, \beta_{K}$ and $\mathbf{g}$ follows from the fact that $T_{-i}$ has more than $K$ distinct elements. ${ }^{20}$

For each $t_{i} \in T_{i}$ and $k=1,2, \ldots, K$, set

$$
w_{k}\left(t_{i}\right)=\min _{\bar{t}_{i}^{\prime} \in T_{i} \backslash U_{k}} d\left(t_{i}, \bar{t}_{i}\right)
$$

and

$$
\alpha_{k}\left(t_{i}\right)=\frac{w_{k}\left(t_{i}\right)}{\sum_{\ell=1}^{K} w_{\ell}\left(t_{i}\right)} .
$$

This is well defined because for each $t_{i} \in T_{i}$, there exists at least one $\ell$ such that $t_{i} \in U_{\ell}$ and therefore $w_{\ell}\left(t_{i}\right)>0$ and $\sum_{\ell=1}^{K} w_{\ell}\left(t_{i}\right)>0$.

We now define $\bar{b}_{i}: T_{i} \rightarrow \mathcal{M}\left(T_{-i}\right)$ by setting

$$
\bar{b}_{i}\left(t_{i}\right)=\sum_{k=1}^{K} \alpha_{k}\left(t_{i}\right) \beta_{k}
$$

for any $t_{i} \in T_{i}$. The functions $w_{k}$ and $\alpha_{k}$ are obviously continuous. Therefore $\bar{b}_{i} \in \mathcal{C}\left(T_{i}, \mathcal{M}\left(T_{-i}\right)\right)$. Moreover, for any $t_{i} \in T_{i}$ and $k=1, \ldots, K, \alpha_{k}\left(t_{i}\right)>0$ implies $t_{i} \in U_{k}$ and hence $\rho\left(\beta_{k}, b_{i}\left(t_{i}\right)\right)<$ $\frac{\eta}{2}$. From using (17) with $\mathcal{L}=\{1, \ldots, K\}, \beta(k)=\beta_{k}$, and $\hat{\beta}(k)=b_{i}\left(t_{i}\right), k=1, \ldots, K$, it follows

\footnotetext{
${ }^{20}$ To see this, observe that, since $T_{-i}$ has infinitely many elements, there exist $K$ distinct elements $t_{-i}^{1}, \ldots, t_{-i}^{K}$ of $T_{-i}$ with open neighbourhoods $B^{1}, \ldots, B^{K}$ that are disjoint. By Urysohn's lemma, there exist continuous functions $g^{k}: T_{-i} \rightarrow[0,1], k=1,2, \ldots K$, such that for each $k, g^{k}\left(t_{-i}^{k}\right)=1$ and $g^{k}\left(t_{-i}\right)=0$ for $t_{-i} \notin B^{k}$. We write $\mathbf{g}=\left(g^{k}\right)_{k=1}^{K}$.

Select measures $\beta_{1}^{0}, \ldots, \beta_{K}^{0}$ in $\mathcal{M}\left(T_{-i}\right)$ such that, for $k=1, \ldots, K, \rho\left(\beta_{k^{\prime}}^{0} b_{i}\left(\bar{t}_{i}\right)\right)<\frac{\eta}{2}$ for some $\bar{t}_{i} \in U_{k}$. If the vectors $\int_{T_{-i}} \mathbf{g}\left(t_{-i}\right) d \beta_{k}^{0}\left(t_{-i}\right), k=1, \ldots, K$, are linearly independent, set $\beta_{k}=\beta_{k}^{0}$ for $k=1, \ldots, K$. If the vectors $\int_{T_{-i}} \mathbf{g}\left(t_{-i}\right) d \beta_{k}^{0}\left(t_{-i}\right), k=1, \ldots, K$, are linearly dependent, set $\beta_{k}=(1-\varepsilon) \beta_{k}^{0}+\varepsilon \delta_{t_{-i}^{k}}$ for $k=1, \ldots, K$, where, for each $k, \delta_{t_{-i}^{k}}$ is the degenerate measure with unit mass at $t_{-i}^{k}$ and $\varepsilon>0$ is sufficiently small so that $\rho\left(\beta_{k}, b_{i}\left(\bar{t}_{i}\right)\right)<\frac{\eta}{2}$ for all $k$. If $\varepsilon$ is chosen not to be an eigenvalue of the matrix $\left(\int_{T_{-i}} \mathbf{g}\left(t_{-i}\right) d \beta_{k}^{0}\left(t_{-i}\right)\right)_{k=1}^{K}$, the vectors $\int_{T_{-i}} \mathbf{g}\left(t_{-i}\right) d \beta_{k}^{0}\left(t_{-i}\right), k=1, \ldots, K$, are linearly independent.
} 
that

$$
\begin{aligned}
\rho\left(\bar{b}_{i}\left(t_{i}\right), b_{i}\left(t_{i}\right)\right) & =\rho\left(\sum_{k=1}^{K} \alpha_{k}\left(t_{i}\right) \beta_{k}, b_{i}\left(t_{i}\right)\right) \\
& \leq \sum_{k=1}^{K} \alpha_{k}\left(t_{i}\right) \rho\left(\beta_{k}, b_{i}\left(t_{i}\right)\right) \\
& <\sum_{k=1}^{K} \alpha_{k}\left(t_{i}\right)\left[\inf _{\bar{t}_{i} \in U_{k}} \rho\left(\beta_{k}, b_{i}\left(\bar{t}_{i}\right)\right)+\frac{\eta}{2}\right] \\
& <\eta,
\end{aligned}
$$

uniformly in $t_{i}$, which establishes (22). In the derivation for (27), the last inequality follows because $\beta_{k}$ had been chosen so that $\rho\left(\beta_{k}, b_{i}\left(\bar{t}_{i}\right)\right)<\frac{\eta}{2}$ for some $\bar{t}_{i} \in U_{k}$; the last inequality but one follows from (24) and the triangle inequality.

It remains to be shown that $\bar{b}_{i} \in G_{\varepsilon}$. For this purpose, consider any $t_{i} \in T_{i}$ and $\mu \in$ $\mathcal{M}\left(T_{i}\right)$, and suppose that

$$
\varphi_{\bar{b}_{i}}\left(\delta_{t_{i}}\right)=\varphi_{\bar{b}_{i}}(\mu)
$$

By construction,

$$
\varphi_{\bar{b}_{i}}\left(\delta_{t_{i}}\right)=\bar{b}_{i}\left(t_{i}\right)=\sum_{k=1}^{K} \alpha_{k}\left(t_{i}\right) \beta_{k}
$$

and

$$
\varphi_{\bar{b}_{i}}(\mu)=\int_{T_{i}} \bar{b}_{i}\left(\bar{t}_{i}\right) \mu\left(d \bar{t}_{i}\right)=\sum_{k=1}^{K} \int_{T_{i}} \alpha_{k}\left(\bar{t}_{i}\right) \beta_{k} \mu\left(d \bar{t}_{i}\right) .
$$

Thus, $\varphi_{\bar{b}_{i}}\left(\delta_{t_{i}}\right)=\varphi_{\bar{b}_{i}}(\mu)$ implies

$$
\sum_{k=1}^{K} \alpha_{k}\left(t_{i}\right) \beta_{k}=\sum_{k=1}^{K} \int_{T_{i}} \alpha_{k}\left(\bar{t}_{i}\right) \beta_{k} \mu\left(d \bar{t}_{i}\right)
$$

For the integrals in (25), we therefore obtain

$$
\begin{aligned}
\sum_{k=1}^{K} \alpha_{k}\left(t_{i}\right) \int_{T_{-i}} \mathbf{g}\left(t_{-i}\right) d \beta_{k}\left(t_{-i}\right) & =\sum_{k=1}^{K} \int_{T_{i}} \alpha_{k}\left(\bar{t}_{i}\right) \int_{T_{-i}} \mathbf{g}\left(\bar{t}_{-i}\right) \beta_{k}\left(d \bar{t}_{-i}\right) \mu\left(d \bar{t}_{i}\right) \\
& =\sum_{k=1}^{K} \int_{T_{i}} \alpha_{k}\left(\bar{t}_{i}\right) \int_{T_{-i}} \mathbf{g}\left(\bar{t}_{-i}\right) \mu\left(d \bar{t}_{i}\right) \beta_{k}\left(d \bar{t}_{-i}\right)
\end{aligned}
$$

and hence,

$$
\sum_{k=1}^{K}\left[\alpha_{k}\left(t_{i}\right)-\int \alpha_{k}\left(\bar{t}_{i}\right) \mu\left(d \bar{t}_{i}\right)\right] \int \mathbf{g}\left(t_{-i}\right) \beta_{k}\left(d t_{-i}\right)=0
$$


Because the integrals $\int_{T_{-i}} \mathbf{g}\left(t_{-i}\right) d \beta_{k}\left(t_{-i}\right), k=1, \ldots, K$, are linearly independent, equation (28) implies that

$$
\alpha_{k}\left(t_{i}\right)-\int \alpha_{k}\left(\bar{t}_{i}\right) \mu\left(d \bar{t}_{i}\right)=0
$$

for all $k$. For any $k$, therefore, $\alpha_{k}\left(t_{i}\right)=0$ implies $\int \alpha_{k}\left(\bar{t}_{i}\right) \mu\left(d \bar{t}_{i}\right)=0$. Since $\alpha_{k}\left(t_{i}\right)=0$ if $t_{i} \notin U_{k}$ and $\alpha_{k}\left(\bar{t}_{i}\right)>0$ if $\bar{t}_{i} \in U_{k}$, it follows that, if $t_{i} \notin U_{k}$, then $\mu\left(U_{k}\right)=0$. Thus, the measure $\mu$ must be concentrated on the union of the sets $U_{k}$ that contain $t_{i}$. By (23), any one of these sets, and therefore their union, is contained in $B_{\zeta}\left(t_{i}\right)$, the open $\zeta$-ball around $t_{i}$. Thus, $\mu\left(B_{\zeta}\left(t_{i}\right)\right)=1$. It follows that $d\left(\delta_{t_{i}}, \mu\right)<\zeta$. Since $\zeta<\varepsilon$, we infer that $\bar{b}_{i} \in G_{\varepsilon}$. Claim 3 is thereby established.

The first statement of the theorem follows from Claims $1-3$.

Claim 4: If $\mathcal{M}\left(T_{-i}\right)$ is complete, $\mathcal{E}^{*}\left(T_{i}, \mathcal{M}\left(T_{-i}\right)\right)$ is dense in $\mathcal{C}\left(T_{i}, \mathcal{M}\left(T_{-i}\right)\right)$.

Proof. If $\mathcal{M}\left(T_{-i}\right)$ is complete, $\mathcal{C}\left(T_{i}, \mathcal{M}\left(T_{-i}\right)\right)$ is also a complete metric space ${ }^{21}$ and therefore a Baire space. The claim thus follows from the first statement of the theorem.

Above we noted that the metrics $\rho^{w}$ and $\rho^{T V}$ for the weak* topology and the totalvariation topology on $\mathcal{M}\left(T_{-i}\right)$ both satisfy the convexity assumption of Theorem 2.4. By standard arguments, in both topologies, $\mathcal{M}\left(T_{-i}\right)$ is also complete. ${ }^{22}$ Thus, with both topologies, $\mathcal{E}^{*}\left(T_{i}, \mathcal{M}\left(T_{-i}\right)\right)$ is also dense in $\mathcal{C}\left(T_{i}, \mathcal{M}\left(T_{-i}\right)\right)$.

\section{Full Surplus Extraction in Universal-Type-Space Models}

\subsection{The Universal Type Space}

We now extend our analysis to the $\Theta$-based universal type space, where $\Theta=\prod_{i=1}^{I} \Theta_{i}$ and $\Theta_{1}, . ., \Theta_{I}$ are compact metric spaces of payoff parameters for agents $1, . ., I$. We restrict our analysis to the special case where each agent $i$ knows his own basic type $t_{i}$ and his own beliefs of different orders.

For $i=1, \ldots, I$, define sets $X_{i}^{0}, X_{i}^{1}, \ldots$ inductively by setting

$$
X_{i}^{0}=\Theta_{-i}, X_{i}^{1}=\Theta_{-i} \times \mathcal{M}\left(X_{-i}^{0}\right),
$$

\footnotetext{
${ }^{21}$ See, e.g., Aliprantis and Border (2007), p. 74.

${ }^{22}$ For the weak ${ }^{*}$ topology, it suffices to note that, because $T_{-i}$ is compact, $\mathcal{M}\left(T_{-i}\right)$ is also compact and hence complete; see Theorem 6.5 in Parthasarathy (1967). For the total-variation topology, see Dunford and Schwartz (1988), p. 161.
} 
and, for any $n \geq 2$,

$X_{i}^{n}=\left\{\left(\theta_{-i}, \mu_{-i}^{1}, \ldots \mu_{-i}^{n}\right) \in \Theta_{-i} \times \mathcal{M}\left(X_{-i}^{0}\right) \ldots \times \mathcal{M}\left(X_{-i}^{n-1}\right): \operatorname{marg}_{X_{-i}^{k-1}} \mu_{-i}^{k}=\mu_{-i}^{k-1}, k=2, \ldots, n\right\}$.

The $\Theta$-based universal type space of agent $i$ is defined as

$$
U_{i}=\left\{\left(\theta_{i}, \mu_{i}^{1}, \mu_{i}^{2}, \ldots\right) \in \Theta_{i} \times \mathcal{M}\left(X_{i}^{0}\right) \times \mathcal{M}\left(X_{i}^{1}\right) \times \ldots: \operatorname{marg}_{X_{i}^{n-1}} \mu_{i}^{n}=\mu_{i}^{n-1}, n=2,3 \ldots\right\} .
$$

For a typical element

$$
u_{i}=\left(\theta_{i}, \mu_{i}^{1}, \mu_{i}^{2}, \ldots\right)
$$

of $U_{i}, \theta_{i}$ indicates the payoff parameters of agent $i$ and $\mu_{i}^{1}, \mu_{i}^{2}, \ldots$ indicates the hierarchy of the agent's beliefs about the other agents' parameters and the other agents' beliefs of different orders.

To consolidate the hierarchy of beliefs of different orders into a single belief about the other agent's types, the spaces $U_{j}, j \neq i$, of other agents' types must be endowed with a measurable structure. Following Mertens and Zamir (1985), we note that, for any $j$, the space $U_{j}$ is a subspace of the product

$$
\bar{U}_{j}=\Theta_{j} \times \mathcal{M}\left(X_{j}^{0}\right) \times \mathcal{M}\left(X_{j}^{1}\right) \times \ldots
$$

For each $j$, the fact that $\Theta_{j}$ is a compact metric space implies that $X_{j}^{0}=\Theta_{-j}$ is also a compact metric space and so is $X_{-j}^{0}$ as well as $\mathcal{M}\left(X_{-j}^{0}\right)$ when endowed with the weak topology. By a straightforward induction, it follows that, for any $n, X_{j}^{n}$ and $X_{-j}^{n}$ are also compact metric spaces and so is $\mathcal{M}\left(X_{-j}^{n}\right)$ when endowed with the weak topology. Given these topologies and the associated Borel $\sigma$-algebras $\mathcal{B}\left(\Theta_{j}\right), \mathcal{B}\left(\mathcal{M}\left(X_{-j}^{0}\right)\right), \mathcal{B}\left(\mathcal{M}\left(X_{-j}^{1}\right)\right)$, $\mathcal{B}\left(\mathcal{M}\left(X_{-j}^{2}\right)\right)$ on the factors of the product in (34), we write $\mathcal{B}\left(\bar{U}_{j}\right)$ for the product $\sigma$-algebra on $\bar{U}_{j}$ and

$$
\mathcal{B}\left(U_{j}\right):=\left\{B \subset U_{j} \mid B \in \mathcal{B}\left(\bar{U}_{i}\right)\right\}
$$

for the induced $\sigma$-algebra on $U_{j}$. We also write $\mathcal{B}\left(U_{-i}\right)$ for the product $\sigma$-algebra on $U_{-i}=$ $\prod_{j \neq i} U_{j}$ and $\mathcal{M}\left(U_{-i}\right)$ for the set of probability measures on $\left(U_{-i}, \mathcal{B}\left(U_{-i}\right)\right)$.

For any $u_{i}=\left(\theta_{i}, \mu_{i}^{1}, \mu_{i}^{2}, \ldots\right) \in U_{i}$, the consistency condition $\operatorname{marg}_{X_{i}^{n-1}} \mu_{i}^{n}=\mu_{i}^{n-1}, n=$ $2,3 \ldots$ implies that the belief hierarchy $\mu_{i}^{1}, \mu_{i}^{2}, \ldots$ satisfies the conditions of Kolmogorov's extension theorem. Consequently, there exists a unique measure

$$
\beta_{i}\left(u_{i}\right) \in \mathcal{M}\left(U_{-i}\right)
$$


that induces $\mu_{i}^{1}, \mu_{i}^{2}, \ldots$ as marginal distributions on $\Theta_{-i}, X_{-i}^{1}, \ldots$ Upon combining the mappings

$$
u_{i} \rightarrow \beta_{i}\left(u_{i}\right)
$$

and

$$
u_{i} \rightarrow \theta_{i}\left(u_{i}\right):=\operatorname{proj}_{\Theta_{i}}\left(u_{i}\right)
$$

one obtains a bijection

$$
u_{i} \rightarrow\left(\theta_{i}\left(u_{i}\right), \beta_{i}\left(u_{i}\right)\right),
$$

between the universal type space $U_{i}$ of agent $i$ and the space $\Theta_{i} \times \mathcal{M}\left(U_{-i}\right)$ of agent $i^{\prime}$ s payoff characteristics and beliefs.

Mertens and Zamir (1985) have shown that this bijection is actually a homeomorphism if the spaces $U_{1}, \ldots, U_{I}$ are endowed with the product topology. Hellwig (2017a) gives an analogous result for the case where $U_{1}, \ldots, U_{I}$ are endowed with the uniform weak topology of Chen et al. (2010). These findings will play an important role in the context of our genericity results below.

\subsection{The FSE Property in the Universal Type Space}

In the universal type space setting, equilibrium payoffs in a strategic game take the form $\Pi_{i}\left(\theta_{1}\left(u_{i}\right), \beta_{1}\left(u_{1}\right), \ldots, \theta_{I}\left(u_{I}\right), \beta_{I}\left(u_{I}\right)\right)$. The analogue of (10), the condition for (approximately) full surplus extraction, takes the form

$$
\bar{\Pi}_{i}\left(\theta_{i}\left(u_{i}\right), \beta_{i}\left(u_{i}\right)\right)-\varepsilon \leq \min _{n} \int z_{n}^{i}\left(u_{-i}\right) \beta_{i}\left(d u_{-i} \mid u_{i}\right) \leq \bar{\Pi}_{i}\left(\theta_{i}\left(u_{i}\right), \beta_{i}\left(u_{i}\right)\right),
$$

where $\bar{\Pi}_{i}\left(\theta_{i}\left(u_{i}\right), \beta_{i}\left(u_{i}\right)\right)$ is the interim expected value of $\Pi_{i}\left(\theta_{1}\left(u_{i}\right), \beta_{1}\left(u_{1}\right), \ldots, \theta_{I}\left(u_{I}\right), \beta_{I}\left(u_{I}\right)\right)$ under the measure $\beta_{i}\left(u_{i}\right)$ that is given by (37).

Whereas condition (40) is formally similar to condition (2) above, with $\beta_{i}$ as the agent's belief mapping, there is an important difference between them. In the abstract-type-space setting, the FSE property was defined as a property of belief functions, and the question was what can be said about the set of belief functions with this property. In the universaltype-space setting, there is only one belief function $\beta_{i}$, which is fixed and cannot be varied without losing the interpretation of the universal type space as a space of payoff characteristics and belief hierarchies.

Moreover, when considered as a function on $U_{i}$ the pair of mappings $\left(\theta_{i}(\cdot), \beta_{i}(\cdot)\right)$ does not satisfy the generalized McAfee-Reny condition for full surplus extraction. In fact, 
given the product structure of both the domain and the range $\Theta_{i} \times \mathcal{M}\left(U_{-i}\right)$ of the mapping $u_{i} \rightarrow\left(\theta_{i}\left(u_{i}\right), \beta_{i}\left(u_{i}\right)\right)$, any belief in $\mathcal{M}\left(U_{-i}\right)$ can be paired with any set of payoff parameters in $\Theta_{i}$. At the level of the universal type space as a whole, the FSE property and the FSE* property fail to hold.

However, these properties can be obtained as properties of subsets of the universal type space. We say that a set $S_{i} \subset U_{i}$ has the FSE* property if the restriction to $S_{i}$ of the mapping (39) has the FSE ${ }^{*}$ property, i.e. if, for every $\varepsilon>0$ and every continuous payoff function $\bar{\Pi}_{i}$ from $\Theta_{i} \times \mathcal{M}\left(U_{-i}\right)$ to $\mathbb{R}_{+}$, there exist a system $z_{1}^{i}, \ldots, z_{N_{i}}^{i}$ of participation fee schedules for agent $i$ such that (40) holds for all $u_{i} \in S_{i}$. We also say that a set $S_{i} \subset U_{i}$ has the FSE property if the restriction to $S_{i}$ of the mapping (37) has the FSE property, i.e. if, for every $\varepsilon>0$ and every continuous payoff function $\bar{\Pi}_{i}$ from $S_{i}$ to $\mathbb{R}_{+}$, there exist a system $z_{1}^{i}, \ldots, z_{N_{i}}^{i}$ of participation fee schedules for agent $i$ such that for all $u_{i} \in S_{i}$,

$$
\bar{\Pi}_{i}\left(u_{i}\right)-\varepsilon \leq \min _{n} \int z_{n}^{i}\left(u_{-i}\right) \beta_{i}\left(d u_{-i} \mid u_{i}\right) \leq \bar{\Pi}_{i}\left(u_{i}\right)
$$

From Theorems 2.1 and 2.2 and the fact that the mapping (39) is a bijection, we immediately obtain:

REMARK 3.1 Let $U_{1}, \ldots, U_{I}$ be metric spaces and assume that the mapping (37) is continuous. A compact set $S_{i} \subset U_{i}$ has the FSE property if and only if it has the FSE* property. Both properties hold if and only if the restriction to $S_{i}$ of the bijection (37) satisfies the McAfee-Reny condition, i.e., if and only if, for all $\bar{u}_{i} \in S_{i}$ and every probability measure $\mu_{i}$ on $S_{i}$,

$$
\varphi_{\beta_{i}}\left(\mu_{i}\right)=\varphi_{\beta_{i}}\left(\delta_{\bar{u}_{i}}\right) \text { implies } \mu_{i}=\delta_{\bar{u}_{i}}
$$

where $\delta_{\bar{u}_{i}}$ is the degenerate measure that assigns all mass to the singleton $\left\{\delta_{\bar{u}_{i}}\right\}$ and $\varphi_{\beta_{i}}\left(\mu_{i}\right)$ is defined to that

$$
\varphi_{\beta_{i}}\left(B \mid \mu_{i}\right):=\int_{S_{i}} \beta_{i}\left(B \mid u_{i}\right) \mu_{i}\left(d u_{i}\right)
$$

for all $B \in \mathcal{B}\left(U_{-i}\right)$.

Given that the FSE and FSE* properties of a compact subset of the universal type space are equivalent, from now on, we drop the distinction and refer only to the FSE property.

For an epistemic interpretation of this property, we introduce the notion of an information base for a subset of the universal type space. For any compact set $S_{i} \subset U_{i}$, a compact 
metric space $T_{i}$ and a continuous mapping $\psi_{i}: T_{i} \rightarrow U_{i}$ are said to provide an information base for $S_{i}$ if $S_{i}$ is the range of $\psi_{i}{ }^{23}$ The following proposition relates the FSE property of a set $S_{i} \subset U_{i}$ to the properties of of any information base $\left(T_{i}, \psi_{i}\right)$ of $S_{i}{ }^{24}$

Proposition 3.2 Assume that $U_{1}, \ldots, U_{I}$ are metric spaces and that the mapping (37) is continuous. A compact set $S_{i} \subset U_{i}$ has the FSE property if and only if, for every information base $\left(T_{i}, \psi_{i}\right)$ of $S_{i}$, the mapping

$$
\left(\theta_{i}^{*}(\cdot), \beta_{i}^{*}(\cdot)\right)=\left(\theta_{i}(\cdot), \beta_{i}(\cdot)\right) \circ \psi_{i}
$$

from $T_{i}$ to $\Theta_{i} \times \mathcal{M}\left(U_{-i}\right)$, which is the composition of the mapping (39) with the mapping $\psi_{i}$, has the FSE* property.

Proof. The "only if" part of the proposition is trivial because any compact set $S_{i} \subset U_{i}$, together with the identity mapping, is an information base for itself. For the "if" part, consider any compact metric space $T_{i}$ and any continuous function $\psi_{i}$ from $T_{i}$ to $U_{i}$ such that $\psi_{i}\left(T_{i}\right)=S_{i}$. The continuity of the pair $\left(\theta_{i}(\cdot), \beta_{i}(\cdot)\right)$ implies that the composition $\left(\theta_{i}^{*}(\cdot), \beta_{i}^{*}(\cdot)\right)=\left(\theta_{i}(\cdot), \beta_{i}(\cdot)\right) \circ \psi_{i}$ is continuous. By Theorem 2.2, $\left(\theta_{i}^{*}(\cdot), \beta_{i}^{*}(\cdot)\right)$ has the FSE $^{*}$ property if and only if it satisfies the generalized McAfee-Reny condition. Thus, we must show that the implication

$$
\varphi_{\beta_{i}^{*}}(\mu)=\varphi_{\beta_{i}^{*}}\left(\delta_{\bar{t}_{i}}\right) \text { implies } \mu \circ\left(\theta_{i}^{*}(\cdot), \beta_{i}^{*}(\cdot)\right)^{-1}=\delta_{\left(\theta_{i}^{*}\left(\bar{t}_{i}\right), \beta_{i}^{*}\left(\bar{t}_{i}\right)\right)}
$$

holds for every $\bar{t}_{i} \in T_{i}$ and every probability measure $\mu_{i}$ on $T_{i}$. Let $\bar{t}_{i} \in T_{i}$ and $\mu_{i} \in \mathcal{M}\left(T_{i}\right)$ be such that the premise of $\left.{ }^{* *}\right)$ holds. Let $\bar{u}_{i}=\psi_{i}\left(\bar{t}_{i}\right)$ and $\hat{\mu}_{i}=\mu_{i} \circ \psi_{i}^{-1}$. Then obviously

$$
\varphi_{\beta_{i}}\left(\hat{\mu}_{i}\right)=\varphi_{\beta_{i}^{*}}(\mu) \text { and } \varphi_{\beta_{i}}\left(\delta_{\bar{u}_{i}}\right)=\varphi_{\beta_{i}}\left(\delta_{\bar{t}_{i}}\right) \text {, }
$$

\footnotetext{
${ }^{23}$ For an extensive discussion of epistemic interpretations of such a formalism in terms of hard or soft information, see Section 2.4 of Dekel and Siniscalchi (2015). In their analysis, the mapping $\psi$ corresponds to the canonical mapping of an abstract type space $T=\left\{T_{i}, \theta_{i}(.), b_{i}(.)\right\}_{i \in I}$ into the universal type space. See also Dekel, Fudenberg, and Morris (2006), in particular, p. 281.

${ }^{24}$ The participation fee schedules $z_{n}^{i}\left(u_{-i}\right)$ in (40) condition on the other agents' payoff parameters and belief hierarchies, not just on their payoff parameters. It is quite possible for $\beta_{i}$ to satisfy the McAfee-Reny condition and for $\left(\theta_{i}(\cdot), \beta_{i}(\cdot)\right) \circ \psi_{i}$ to satisfy the generalized McAfee-Reny condition even though the function $\operatorname{proj}_{\mathcal{M}\left(\Theta_{-i}\right)} \circ \beta_{i}(\cdot)$ that indicates the agent's first-order beliefs about the other agents' payoff parameters violates the McAfee-Reny condition and $\left(\theta_{i}(\cdot), \operatorname{proj}_{\mathcal{M}\left(\Theta_{-i}\right)} \circ \beta_{i}(\cdot)\right) \circ \hat{\psi}_{i}$ violates the generalized McAfee-Reny condition. In this case, fee schedules that condition on the other agents' belief hierarchies, as well as their payoff parameters, can provide for surplus extraction where fee schedules conditioning on payoff parameters alone cannot. For a detailed discussion and examples, see Gizatulina (2015).
} 
so the premise of $\left.{ }^{* *}\right)$ implies that $\varphi_{\beta_{i}}\left(\hat{\mu}_{i}\right)=\varphi_{\beta_{i}}\left(\delta_{\bar{u}_{i}}\right)$. If $S_{i}$ has the FSE property, then, by Remark 3.1, it follows that $\hat{\mu}_{i}=\delta_{\bar{u}_{i}}$. Therefore,

$$
\mu \circ\left(\theta_{i}^{*}(\cdot), \beta_{i}^{*}(\cdot)\right)^{-1}=\mu \circ \psi_{i}^{-1} \circ\left(\theta_{i}(\cdot), \beta_{i}(\cdot)\right)^{-1}=\delta_{\bar{u}_{i}} \circ\left(\theta_{i}(\cdot), \beta_{i}(\cdot)\right)^{-1}=\delta_{\left(\theta_{i}^{*}\left(\bar{t}_{i}\right), \beta_{i}^{*}\left(\bar{t}_{i}\right)\right)}
$$

where the first equation follows from (41), the second equation from the equation $\hat{\mu}_{i}=\delta_{\bar{u}_{i}}$, and the third equation from the fact that, by the definition of $\bar{u}_{i},\left(\theta_{i}\left(\bar{u}_{i}\right), \beta_{i}\left(\bar{u}_{i}\right)\right)=\psi_{i}\left(\bar{t}_{i}\right)$. The implication $\left(^{* *}\right)$ is thus satisfied and the mapping $\psi_{i}$ thus has the FSE* property if $S_{i}$ has the FSE property.

Proposition 3.2 implies, in particular, that, if a set $S_{i} \subset U_{i}$ has two different information bases $\left(T_{i}^{1}, \psi_{i}^{1}\right),\left(T_{i}^{2}, \psi_{i}^{2}\right)$, then either both of the mappings $\left(\theta_{i}(\cdot), \beta_{i}(\cdot)\right) \circ \psi_{i}^{1}$ and $\left(\theta_{i}(\cdot), \beta_{i}(\cdot)\right) \circ \psi_{i}^{2}$ have the FSE* property or none of them has it. Whereas the notion of information base might look like a device to reintroduce abstract type spaces by the back door, the scope for full surplus extraction is independent of the details of the space $T_{i}$ of information variables and depends only on the range of the mapping

$$
\psi_{i}: T_{i} \rightarrow U_{i}
$$

that maps information variables into pairs of payoff parameters and belief hierarchies. This range, and by implication, the possibility of full surplus extraction, is unaffected under any strategically irrelevant modification of $T_{i}$ such as the introduction of additional information values with associated pairs of payoff parameters and belief hierarchies duplicating some of those that are already in the range of $\psi_{i}$ or a replacement of $\psi_{i}$ by the composition of $\psi_{i}$ with some permutation $p$ of the set of information variables.

\subsection{Genericity of the FSE Property in the Class of Compact Subsets of the Uni- versal Type Space}

The following result shows that the FSE property is generic in the class of compact subsets of the universal type space.

THEOREM 3.3 Assume that $U_{1}, \ldots, U_{I}$ are metric spaces and endow the space of closed subsets of $U_{i}$ with the Hausdorff topology. Assume further that the topology on $\mathcal{M}\left(U_{-i}\right)$ is at least as fine as the topology of weak convergence and is induced by a metric that is a convex function on $\mathcal{M}\left(U_{-i}\right)$ $\times \mathcal{M}\left(U_{-i}\right)$ and that the mapping (39) from $U_{i}$ to $\Theta_{i} \times \mathcal{M}\left(U_{-i}\right)$ is a homeomorphism. Then the 
set of compact subsets of $U_{i}$ that have the FSE property is a residual subset of the class of compact subsets of $U_{i}$.

Theorem 3.3 is our main result concerning the genericity of full surplus extraction in the universal type space. Like Theorem 2.4, Theorem 3.3 does not specify any particular topologies on the spaces that are involved, but merely give qualitative conditions that the topologies must satisfy. The requirement that $U_{-i}$ be a metric space is of course satisfied when the spaces $U_{1}, . ., U_{I}$ have the product topology, as in Mertens and Zamir (1985). In this case, obviously, $\mathcal{M}\left(U_{-i}\right)$ with the topology of weak convergence is also a metric space, and, by the argument given in Section 2.3, the metric can be specified as a convex function on $\mathcal{M}\left(U_{-i}\right) \times \mathcal{M}\left(U_{-i}\right)$. Moreover, as was shown by Mertens and Zamir (1985), the mapping $u_{i} \rightarrow\left(\theta_{i}\left(u_{i}\right), \beta_{i}\left(u_{i}\right)\right)$ defines a homeomorphism between $U_{i}$ and $\Theta_{i} \times \mathcal{M}\left(U_{-i}\right)$, so all the topological requirements of Theorem 3.3 are satisfied.

However, the product topology is not the only topology on the universal type space for which the theorem applies. It also applies if the spaces $U_{1}, . ., U_{I}$ have the uniform strategic topology of Dekel, Fudenberg, and Morris (2006) or the uniform weak topology of Chen, DiTillio, Faingold, and Xiong (2010), which have been proposed to ensure that the minimal $\varepsilon \geq 0$ for which strategies are interim $\varepsilon$-rationalizable depends continuously on their types, a property that Dekel, Fudenberg, and Morris (2006) refer to as lower semicontinuity. ${ }^{25}$ These two topologies actually coincide, and both are metrizable by a metric that defines the distance between two elements $u_{i}^{1}, u_{i}^{2}$ of the universal type space for agent $i$ as the supremum of the distances between the individual components of $u_{i}^{1}$ and $u_{i}^{2}$, the distance between the payoff parameters $\theta_{i}^{1}$ and $\theta_{i}^{2}$, the first-order beliefs $\left(\mu_{i}^{1}\right)^{1}$ and $\left(\mu_{i}^{1}\right)^{2}$, etc. ${ }^{26}$ Given this metric on the universal type spaces $U_{1}, . ., U_{I}$, the topology of weak convergence on $\mathcal{M}\left(U_{-i}\right)$ is specified with reference to the convergence of integrals of bounded continuous real-valued functions on $U_{-i}$ where continuity is defined with in terms of the product topology on $U_{-i}$ that is induced by the uniform weak topology on $U_{j}, j \neq i$.

\footnotetext{
${ }^{25}$ The product topology is too coarse for this purpose. Because, under the product topology, the weight given to higher order beliefs eventually declines as one moves up in the hierarchy of beliefs, the product topology is unsuitable for capturing the continuity properties of strategic behaviour in games such as Rubinstein's e-mail game, in which beliefs of arbitrarily high orders can make a significant difference. See Rubinstein (1989), Dekel, Fudenberg, and Morris (2006), Chen, DiTillio, Faingold, and Xiong (2010).

${ }^{26}$ See Chen et al. $(2010,2012)$.
} 
If the spaces $U_{1}, . ., U_{I}$ are endowed with the uniform weak topology, the induced $\sigma$ algebras are actually larger than the product $\sigma$-algebras, but Hellwig (2017a) and Hellwig (2017b)) show that any set in the $\sigma$-algebra that is induced by the uniform weak topology is measurable by the completion of any measure on the product $\sigma$-algebra. ${ }^{27}$ Any measure on the product $\sigma$-algebra can therefore be uniquely extended to a measure on the $\sigma$-algebra that is induced by the uniform weak topology. Using the notation $\bar{\beta}_{i}\left(u_{i}\right)$ for the extension of $\beta_{i}\left(u_{i}\right)$, Hellwig (2017a) demonstrates that, if $U_{1}, . ., U_{I}$ are endowed with the uniform weak topology, the mapping

$$
u_{i} \rightarrow\left(\theta_{i}\left(u_{i}\right), \bar{\beta}_{i}\left(u_{i}\right)\right)
$$

defines a homeomorphism between the spaces $U_{i}$ and $\Theta_{i} \times \mathcal{M}\left(U_{-i}\right)$ Moreover, the topology of weak convergence on the space $\mathcal{M}\left(U_{-i}\right)$ is metrizable, and is induced by the bounded Lipschitz metric. Because, as we saw in Section 2.3, the bounded Lipschitz metric does satisfy the convexity assumption of Theorem 3.3, this theorem is also applicable when the universal type space is given the uniform weak topology. ${ }^{28}$

The proof of Theorem 3.3 makes use of Proposition 3.2 and of the genericity results in Section 2. We begin with a restatement of Corollary 2.5 for the present setting.

REMARK 3.4 Assume that $T_{i}$ is a compact metric space, that $U_{-i}$ is a metric space, and that the topology on $\mathcal{M}\left(U_{-i}\right)$ is at least as fine as the topology of weak convergence and is induced by a metric that is a convex function on $\mathcal{M}\left(U_{-i}\right) \times \mathcal{M}\left(U_{-i}\right)$. Then the set $\mathcal{E}^{* *}\left(T_{i}, \Theta_{i} \times \mathcal{M}\left(U_{-i}\right)\right)$ of continuous functions from $T_{i}$ to $\Theta_{i} \times \mathcal{M}\left(U_{-i}\right)$ that have the FSE* property is a residual subset of the space $\mathcal{C}\left(T_{i}, \Theta_{i} \times \mathcal{M}\left(U_{-i}\right)\right)$, i.e. $\mathcal{E}^{* *}\left(T_{i}, \Theta_{i} \times \mathcal{M}\left(U_{-i}\right)\right)$ contains a countable intersection of open and dense subsets of $\mathcal{C}\left(T_{i}, \Theta_{i} \times \mathcal{M}\left(U_{-i}\right)\right)$. If the metric space $\Theta_{i} \times \mathcal{M}\left(U_{-i}\right)$ is complete, $\mathcal{E}^{* *}\left(T_{i}, \Theta_{i} \times \mathcal{M}\left(U_{-i}\right)\right)$ is itself dense in $\mathcal{C}\left(T_{i}, \Theta_{i} \times \mathcal{M}\left(U_{-i}\right)\right)$.

${ }^{27}$ Chen, DiTillio, Faingold, and Xiong (2010) claim the opposite, but Chen, diTillio, Faingold, and Xiong (2016) correct this claim and give an example of a subset of $U_{i}$ that is Borel if $U_{i}$ has the uniform weak topology but not, if $U_{i}$ has the product topology. The set in question is analytic and therefore universally measurable, i.e. measurable in the completion of any measure on the product $\sigma$-algebra.

${ }^{28}$ In contrast, Chen and Xiong (2013) assume that the universal type space has the product topology and make essential use of this assumption. With the product topology, the universal type space is separable and, in the topology of weak convergence, the set of priors with finite supports is dense. This fact is crucial For the uniform weak topology on the universal type space, the results of Chen, DiTillio, Faingold, and Xiong (2010) imply that the set of priors with finite supports is not dense. 
Given a compact metric space $T_{i}$, we say that a set $S_{i} \subset U_{i}$ is $T_{i}$-compatible if there exists a continuous function $\psi_{i}: T_{i} \rightarrow U_{i}$ such that the pair $\left(T_{i}, \psi_{i}\right)$ provides an information base for $S_{i}$.

Proposition 3.5 Assume that $U_{1}, \ldots, U_{I}$ are metric spaces and endow the space of closed subsets of $U_{i}$ with the Hausdorff topology. Assume further that the topology on $\mathcal{M}\left(U_{-i}\right)$ is at least as fine as the topology of weak convergence and is induced by a metric that is a convex function on $\mathcal{M}\left(U_{-i}\right) \times \mathcal{M}\left(U_{-i}\right)$ and that the mapping mapping (39) from $U_{i}$ to $\Theta_{i} \times \mathcal{M}\left(U_{-i}\right)$ is a homeomorphism. If $T_{i}$ is a compact metric space, the set of $T_{i}$-compatible subsets of $U_{i}$ that have the FSE property is a residual subset of the set of $T_{i}$-compatible subsets of $U_{i}$.

Proof. Let $T_{i}$ be any compact metric space. Because homeomorphisms map open and dense sets into open and dense sets, Remark 3.4 and the assumption that the mapping (39) from $U_{i}$ to $\Theta_{i} \times \mathcal{M}\left(U_{-i}\right)$ is a homeomorphism imply that the set of functions $\psi_{i}: T_{i} \rightarrow U_{i}$ for which the composition (41) has the $\mathrm{FSE}^{*}$ property is a residual subset of the space $\mathcal{C}\left(T_{i}, U_{i}\right)$.

For any $\psi_{i} \in \mathcal{C}\left(T_{i}, U_{i}\right)$, let $G_{i}\left(T_{i}, \psi_{i}\right) \subset T_{i} \times U_{i}$ be the graph of $\psi_{i}$. Because $T_{i}$ is compact and $\mathcal{C}\left(T_{i}, U_{i}\right)$ has the topology of uniform convergence, the map $\psi_{i} \rightarrow G_{i}\left(T_{i}, \psi_{i}\right)$ is a homeomorphism. ${ }^{29}$ By another application of the fact that homeomorphisms map open and dense sets into open and dense sets, it follows that the graphs $G_{i}\left(T_{i}, \psi_{i}\right)$ of the functions $\psi_{i}: T_{i} \rightarrow U_{i}$ for which the composition (41) has the FSE* property form a residual subset of the class $\left\{G_{i}\left(T_{i}, \psi_{i}\right): \psi_{i} \in \mathcal{C}\left(T_{i}, U_{i}\right)\right\}$. Because, for any $\psi_{i} \in \mathcal{C}\left(T_{i}, U_{i}\right)$, the range $S_{i}\left(T_{i}, \psi_{i}\right)$ of $\psi_{i}$ is the projection of $G_{i}\left(T_{i}, \hat{\psi}_{i}\right)$ to $U_{i}$ and the projection mapping is open and continuous, it follows that the ranges $S_{i}\left(T_{i}, \psi_{i}\right)$ of the functions $\psi_{i} \in \mathcal{C}\left(T_{i}, U_{i}\right)$ for which the composition (41) has the FSE* property form a residual subset of the class $\left\{S_{i}\left(T_{i}, \psi_{i}\right): \psi_{i} \in \mathcal{C}\left(T_{i}, U_{i}\right)\right\}$, i.e. the class of $T_{i}$-compatible subsets of $U_{i}$. Since the ranges $S_{i}\left(T_{i}, \psi_{i}\right)$ of the functions $\psi_{i} \in \mathcal{C}\left(T_{i}, U_{i}\right)$ are just the $T_{i}$-compatible subsets of the universal type space, the proposition follows.

Proof of Theorem 3.3. Let $\mathcal{T}_{i}=\left\{T_{i}^{k}\right\}_{k \in \mathcal{K}}$ be the family of compact metric subsets of the countable product $[0,1]^{\infty}$. For any $k$, let $E_{i}\left(T_{i}^{k}\right)$ be the class of $T_{i}^{k}$-based subsets of $U_{i}$ that have the FSE property. Then

$$
E_{i}\left(\mathcal{T}_{i}\right):=\cup_{k \in \mathcal{K}} E_{i}\left(T_{i}^{k}\right)
$$

\footnotetext{
${ }^{29}$ See Naimpally (1966).
} 
is the class of sets $S_{i} \subset U_{i}$ that have the FSE property and that have an information base $\left(T_{i}, \psi_{i}\right)$ with $T_{i} \in \mathcal{T}_{i}$. We will show that $E_{i}\left(\mathcal{T}_{i}\right)$ contains a countable intersection of open and dense sets of compact subsets of $U_{i}$.

For any $k$, let $E_{i}\left(T_{i}^{k}\right)$ be the class of $T_{i}^{k}$-based subsets of $U_{i}$ that have the FSE property. Proposition 3.5 implies that, for any $k \in \mathcal{K}$, there exists a sequence $\left\{O_{n}^{k}\right\}_{n=1}^{\infty}$ of open and dense sets of compact subsets of $U_{i}$ such that

$$
\begin{gathered}
E_{i}\left(T_{i}^{k}\right) \supset \cap_{n=1}^{\infty} O_{n}^{k} . \\
E_{i}\left(\mathcal{T}_{i}\right) \supset \cup_{k \in \mathcal{K}} \cap_{n=1}^{\infty} O_{n}^{k} .
\end{gathered}
$$

We claim that we also have

$$
\cup_{k \in \mathcal{K}} \cap_{n=1}^{\infty} O_{n}^{k}=\cap_{n=1}^{\infty} \cup_{k \in \mathcal{K}} O_{n}^{k}
$$

and therefore,

$$
E_{i}\left(\mathcal{T}_{i}\right) \supset \cap_{n=1}^{\infty} \cup_{k \in \mathcal{K}} O_{n}^{k}
$$

To prove this claim, we note that, by the argument in the proof of Proposition 3.5, every one of the sets $O_{n}^{k}$ is of the form

$$
O_{n}^{k}=\left\{\operatorname{proj}_{U_{i}} G \mid G \in P_{n}^{k}\right\},
$$

where $P_{n}^{k}$ is a set of graphs $G_{i}\left(T_{i}^{k}, \psi_{i}\right) \subset T_{i}^{k} \times U_{i}$ of functions $\psi_{i} \in \mathcal{C}\left(T_{i}, U_{i}\right)$. For any $k$ and $n$ and any $G \in P_{n}^{k}$,

$$
\operatorname{proj}_{[0,1]^{\infty}} G=T_{i}^{k}
$$

For any $k$ and $k^{\prime} \neq k$ and any $n$ and $n^{\prime}, G \in P_{n}^{k} \operatorname{implies} \operatorname{proj}_{[0,1]^{\infty}} G \neq T_{i}^{k^{\prime}}$ and therefore $G \notin P_{n^{\prime}}^{k^{\prime}}$. Thus, $P_{n}^{k} \cap P_{n^{\prime}}^{k^{\prime}}=\varnothing$ if $k^{\prime} \neq k$. By elementary set theory, it follows that

$$
\cup_{k \in \mathcal{K}} \cap_{n=1}^{\infty} P_{n}^{k}=\cap_{n=1}^{\infty} \cup_{k \in \mathcal{K}} P_{n}^{k}
$$

But then, we also have

$$
\begin{aligned}
\cup_{k \in \mathcal{K}} \cap_{n=1}^{\infty} O_{n}^{k} & =\cup_{k \in \mathcal{K}} \cap_{n=1}^{\infty}\left\{\operatorname{proj}_{U_{i}} G \mid G \in P_{n}^{k}\right\} \\
& =\left\{\operatorname{proj}_{U_{i}} G \mid G \in \cup_{k \in \mathcal{K}} \cap_{n=1}^{\infty} P_{n}^{k}\right\} \\
& =\left\{\operatorname{proj}_{U_{i}} G \mid G \in \cap_{n=1}^{\infty} \cup_{k \in \mathcal{K}} P_{n}^{k}\right\} \\
& =\cap_{n=1}^{\infty} \cup_{k \in \mathcal{K}}\left\{\operatorname{proj}_{U_{i}} G \mid G \in P_{n}^{k}\right\} \\
& =\cap_{n=1}^{\infty} \cup_{k \in \mathcal{K}} O_{n}^{k}
\end{aligned}
$$


which proves (47).

Now, for any $n$, the set $\cup_{k \in \mathcal{K}} O_{n}^{k}$ is open, being a union of open sets. It is also dense in the union $\cup_{k \in \mathcal{K}}\left\{S_{i}\left(T_{i}^{k}, \psi_{i}\right): \psi_{i} \in \mathcal{C}\left(T_{i}^{k}, U_{i}\right)\right\}$ because, by the denseness of the sets $O_{n}^{k}$ for $k \in \mathcal{K}$, for any $k^{\prime} \in \mathcal{K}$ and $\hat{\psi}_{i} \in \mathcal{C}\left(T_{i}^{k^{\prime}}, U_{i}\right)$, any neighbourhood of $S_{i}\left(T_{i}^{k^{\prime}}, \hat{\psi}_{i}\right)$ intersects $O_{n}^{k^{\prime}}$ and therefore $\cup_{k \in \mathcal{K}} O_{n}^{k}$.

To complete the argument, we note that any compact set $S_{i} \subset U_{i}$ is homeomorphic to a compact subset of the product $[0,1]^{\infty}$. ${ }^{30}$ The union $\cup_{k \in \mathcal{K}}\left\{S_{i}\left(T_{i}^{k}, \psi_{i}\right): \psi_{i} \in \mathcal{C}\left(T_{i}^{k}, U_{i}\right)\right\}$ is therefore just equal to the set of compact subsets of $U_{i}$. Residualness of the FSE property in the union of $T_{i}^{k}$-based subsets of $U_{i}, k \in \mathcal{K}$, is equivalent to residualness in the class of compact subsets of $U_{i}$.

\subsection{Genericity of the FSE Property in the Space of Common Priors on the Uni- versal Type Space}

We finally provide a universal type space analogue of Theorem 2.7. Let $\mathcal{M}^{*}(U)$ of common priors, the set of probability measures on $U$ such that, for each $i$, the function $\beta_{i}(\cdot)$ that is given by Kolmogorov's extension theorem is a regular conditional distribution for $u_{-i}$ given $u_{i}$. Consider the set $\mathcal{M}_{f}^{c}(U) \subset \mathcal{M}^{*}(U)$ such that, for any $\mu \in \mathcal{M}_{f}^{c}(U)$ and any $i$, the support of the marginal distribution $v_{i}(\mu)$ is a compact set. We say that $\mu \in \mathcal{M}_{f}^{c}(U)$ has the FSE property if and only if, for every $i$ the support of the marginal distribution $v_{i}(\mu)$ has the FSE property.

Given Theorem 3.3, the same argument that was used to derive Theorem 2.7 from Theorem 2.4 now yields a genericity result in the space of priors on the universal type space.

THEOREM 3.6 Assume that $U_{1}, \ldots, U_{I}$ are metric spaces. For each $i$, endow the space of closed subsets of $U_{i}$ with the Hausdorff topology. Assume that the topology on $\mathcal{M}\left(U_{-i}\right)$ is at least as fine as the topology of weak convergence and is induced by a metric that is a convex function on $\mathcal{M}\left(U_{-i}\right) \times \mathcal{M}\left(U_{-i}\right)$ and, finally, that the mapping (39) from $U_{i}$ to $\Theta_{i} \times \mathcal{M}\left(U_{-i}\right)$ is a homeomorphism. If $\mathcal{M}_{f}^{c}(U)$ is endowed with the coarsest topology under which the mappings from priors to the supports of marginal distributions on the spaces $U_{i}, i=1, \ldots, I$, are continuous, then the set $\mathcal{F}(U)$ of priors in $\mathcal{M}_{f}^{c}(U)$ that have the FSE property is a residual subset of $\mathcal{M}_{f}^{c}(U)$.

Proof. For any $i$, let $\mathcal{F}_{i}(U)$ be the set of priors $\mu \in \mathcal{M}_{f}^{c}(U)$ such that the support of the

\footnotetext{
${ }^{30}$ See, e.g., Engelking (1989), p. 260.
} 
marginal distribution $v_{i}(\mu)$ has the FSE property. By the argument given in the proof of Theorem 2.7, it suffices to prove that each one of the sets $\mathcal{F}_{i}(U), i=1, \ldots, I$, is residual in $\mathcal{M}_{f}^{c}(U)$. For each $i$, the supports of the marginal distributions $v_{i}(\mu), \mu \in \mathcal{M}_{f}^{c}(U)$, belong to the class $\mathcal{S}_{i}$ of compact subsets of $U_{i}$.

The mapping $\mu \rightarrow \operatorname{supp} v_{i}(\mu)$ is in fact the composition of the mapping

$$
\mu \rightarrow\left(\operatorname{supp} v_{1}(\mu), \ldots, \operatorname{supp} v_{I}(\mu)\right)
$$

from $\mathcal{M}_{f}^{c}(U)$ to the product $\mathcal{S}_{1} \times \ldots \times \mathcal{S}_{I}$ with the projection from $\mathcal{S}_{1} \times \ldots \times \mathcal{S}_{I}$ to $\mathcal{S}_{i}$. For the given topology on $\mathcal{M}_{f}^{c}(U)$, the mapping (49) is open and continuous. Because the projection from $\mathcal{S}_{1} \times \ldots \times \mathcal{S}_{I}$ to $\mathcal{S}_{i}$ is also open and continuous, it follows that the mapping $\mu \rightarrow \operatorname{supp} v_{i}(\mu)$ is also open and continuous. The theorem now follows from Remark 3.4 and the observation that open and dense sets are preserved under continuous and open mappings and their inverses.

\section{Relation to the Literature}

\subsection{Relation to Heifetz and Neeman (2006)}

The thrust of our results runs counter to that of Heifetz and Neeman (2006). They consider families of incomplete-information models (7) that are consistent with common priors and study the genericity of the full surplus extraction property within the set $\mathcal{P}$ of common priors for the models in a given family. Under the additional assumption that the family of models is what they call "closed under finite unions", they show that $\mathcal{P}$ is a convex set and that any prior of the form $F=\sum_{j=1}^{J} \alpha_{j} F^{j}$ with $\alpha_{j}>0$ and $F^{j} \in \mathcal{P}$ for all $j$ has the BDP property if and only if each of the distributions $F^{j}$ has the BDP property. Because the BDP property is necessary for full surplus extraction, they conclude that, unless all incomplete-information models $\mathcal{T}^{k}, k \in K$, have BDP priors, the set of priors that do not admit full surplus extraction is geometrically and measure-theoretically generic in $\mathcal{P}$.

The difference between our results and those of Heifetz and Neeman (2006) is not only a matter of topological versus geometric or measure-theoretic genericity but also one of genericity in the full space versus genericity in the set of priors for the models in a given family. This restriction begs the question what can be said about the set of families for which they obtain non-genericity of full surplus extraction relative to the set of all families of incomplete-information models that are closed under finite unions. 
The requirement that the family be closed under finite unions can actually be quite restrictive. In related work (Gizatulina and Hellwig (2013)), we show that, if, for any $i$, the type sets $T_{i}^{k}$ of agent $i$ in different models in the family are subsets of a complete separable metric space and if they have non-empty interiors, then a family of models that is closed under finite unions is at most countable. As a consequence of Theorem 2.7, one can then show that, for a given sequence of such type sets, full surplus extraction can be obtained for all models in a residual set of families. The set of families to which the Heifetz and Neeman (2006) analysis applies, i.e. the set of families with at least one member for which full surplus extraction cannot be obtained, is itself sparse in the set of all families of models with the given family of type spaces.

\subsection{Relation to Chen and Xiong (2013)}

Chen and Xiong (2013) also study the genericity of (almost) full surplus extraction. They consider common priors on the universal type space and define approximately full surplus extraction in terms of expected surplus, rather than type by type. They endow the universal type space with the product topology and the space of common priors with the topology of weak convergence. For a special class of allocation problems and payoff functions in which it is feasible to exclude agents on an individual basis, they show that a residual set of priors admits approximately full surplus extraction.

The argument is, briefly, the following: Since we know from Crémer and McLean (1988) that full surplus extraction is generic in the set of priors with finite supports, with a topology in which priors with finite supports are dense, it follows that every prior can be approximated by priors that admit full surplus extraction. Residualness follows by showing that, for every $\varepsilon>0$, the set of priors that extract all but $\varepsilon$ of the expected surplus is open. For this purpose, Chen and Xiong (2013) take an FSE prior with a sufficiently large finite support, extend the Crémer-McLean payment function to a continuous function on the entire space and reduce each agent's payment by some $\eta>0$. For every prior in a neighbourhood of the FSE prior, the resulting mechanism extracts all but $2 \eta$ on a set of probability $1-\eta$. On the remaining set of types, an agent's expected net payoff may be large (but bounded) or negative. If it is negative, the agent is excluded; exclusion involves a loss of surplus but as long as the amount is bounded and the other agents' incentives are unaffected, this loss does not matter because its probability is small.

The analysis of Chen and Xiong (2013) does not actually depend on their working with 
the universal type space but goes through with abstract (Harsanyi) type spaces as well. However, the analysis does depend on (i) the assumption that agents can be excluded on an individual basis, (ii) the definition of approximately full surplus extraction in terms of expected values, rather than type by type, and (iii) reliance on a topology in which the priors with finite supports are dense.

If there is no scope for such an exclusion of individuals the argument of Chen and Xiong (2013) cannot be used. ${ }^{31}$ An example would the problem of providing a nonexcludable public good when there are interim participation constraints. Whereas it is well known that, with independent private values, in this problem efficiency cannot be achieved, ${ }^{32}$ our results in this paper suggest that, with correlated private values, it is generically possible to achieve $\varepsilon$-efficiency, where $\varepsilon>0$ may be taken to be arbitrarily small. In this context, the argument of Chen and Xiong (2013) cannot be used because one cannot exclude agents individually. One can "exclude" all participants jointly by not providing the public good at all, but if one uses this kind of exclusion to discipline one agent, there may be harmful side effects on the incentives of other agents.

Because exclusion may significantly reduce the surplus that is achieved, in the approach of Chen and Xiong (2013), approximately full surplus extraction is not to be expected type by type, but only in terms of expected values. This is why full surplus extraction in the sense of Chen and Xiong has to be defined in terms of priors, rather than type by type, as in (5). Given that approximately full surplus extraction is defined in terms of expected values only, Chen and Xiong (2013) do not need the generalized McAfee-Reny condition, as agents who make for a violation of this condition can simply be excluded. Without exclusion, and with a notion of (approximately) full surplus extraction type by type, the generalized McAfee-Reny condition is necessary.

In abstract type spaces, the presumption that finite-support priors are dense excludes the possibility that beliefs over other agents types might be topologized by the total variation norm. In the universal type space, this presumption excludes the possibility of imposing the uniform weak or the uniform strategic topology. In contrast, by working directly with the (generalized) McAfee-Reny condition, we forego the need to work with finite approximations so our results leave more freedom for the specification of the topologies on beliefs.

\footnotetext{
${ }^{31}$ More precisely, as they point out, the mechanism designer must be able to impose an outcome at which agent $i$ 's net payoff is zero no matter what types the other agents may have.

${ }^{32}$ See Güth and Hellwig (1986), Rob (1989) and Mailath and Postlewaite (1990).
} 


\section{Concluding Remarks}

Our paper greatly extends the domain of Crémer and McLean (1988) finding that in models with correlated types, it is generically possible to design incentive mechanisms so as to extract all the available surplus. Whereas Crémer and McLean (1988) established this finding for models with finite type sets, we allow agents' type sets to be arbitrary, e.g. infinite-dimensional, compact spaces.

The key to our analysis is a new mathematical result showing that the condition that McAfee and Reny (1992) established as being necessary and sufficient for approximately full surplus extraction in naive type spaces is satisfied by a residual set of belief functions, i.e. of continuous functions mapping an agent's types to his beliefs about the possible constellations of other agents' types. In arbitrary abstract type types, the McAfee-Reny condition may be stronger than necessary but then its genericity implies that the gener-

alized McAfee-Reny condition, which is necessary and sufficient for approximately full surplus extraction, is also satisfied generically.

In the universal type space, we study the possibility of surplus extraction as a property of subsets of the universal type space that is obtained if the restriction of the Kolmorogov mapping to the subset in question satisfies the McAfee-Reny condition. Relying on our results for abstract type spaces, we find that the set of subsets of the universal type space that admit full surplus extraction is residual in the class of compact subsets of the universal type space.

Our genericity results do not rely on any particular topology. We only require that the set of an agent's types be a compact metric space and that the topology on the space of an agent's beliefs be metrizable in a certain way.

Our separate analyses of the genericity of full surplus extraction in abstract and universal type spaces raise the question whether the two sets of results can be linked. The universal type space takes its name from the proposition, proved by Mertens and Zamir (1985), that every abstract type space can be embedded in it. It seems natural to suppose that the image of this embedding in the universal type space is a set admitting full surplus extraction if and only the abstract-type-space model does. However, except for the case where the universal type space has the product topology and all beliefs have the relevant topology of weak convergence, it is not clear how the topologies on the different spaces are matched. In the abstract-type-space model, one might impose a topology so that, the mapping to the universal type space is continuous, but then the question is whether this 
topology has an interpretation of its own in the abstract-type-space model itself. Obtaining a better understanding of the relation between the different topologies on the different spaces and, by implication, of the relation between different genericity results is a problem for future research.

\section{Appendix A Generalizing the McAfee-Reny Result: Proof Sketch for Theorem 2.1}

The proof of Theorem 2.1 is by and large the same as the proof of Theorem 2 in McAfee and Reny (1992), with due changes to account for the fact that $T_{i}$ is an arbitrary compact metric space, rather than the unit interval, and for the fact that beliefs need not have densities. Therefore we will not go into all the details but merely indicate where and how the argument of McAfee and Reny must be adapted.

In the analysis of McAfee and Reny (1992), a special role is played by what they call the set of " $(\varepsilon, \delta)$-u-shaped functions at $t_{i 0}$ ". In the present, more general setting, an $(\varepsilon, \delta)$ u-shaped function at $t_{i 0}$ is a function $u \in \mathcal{C}\left(T_{i}\right)$ such that

(i) $u\left(t_{i}\right) \geq 0$ for all $t_{i} \in T_{i}$,

(ii) $u\left(t_{i 0}\right) \leq \varepsilon$, and

(iii) $u\left(t_{i}\right) \geq 1$ for all $t_{i} \in T_{i} \backslash B^{\delta}\left(t_{i 0}\right)$, where $B^{\delta}\left(t_{i 0}\right)$ is the closed $\delta$-ball around $t_{i 0}$.

The set of such $(\varepsilon, \delta)$-u-shaped function at $t_{i 0}$ is denoted as $U\left(\varepsilon, \delta, t_{i 0}\right)$. This set plays a key role in the following auxiliary result, which extends Theorem 1 of McAfee and Reny (1992).

Proposition A.1 Suppose that a set $A \subset \mathcal{C}\left(T_{i}\right)$ satisfies:

(i) $A$ is closed under addition and under multiplication by a positive scalar.

(ii) For any $N, x_{1}, \ldots, x_{N} \in A$ implies $y \in A$, where $y$ is defined by setting $y\left(t_{i}\right)=\min \left(x_{1}\left(t_{i}\right), \ldots, x_{N}\left(t_{i}\right)\right)$ for any $t_{i} \in T_{i}$.

(iii) $1,-1 \in A$

(iv) For all $\varepsilon>0, \delta>0$, and every $t_{i 0} \in T_{i}, U\left(\varepsilon, \delta, t_{i 0}\right) \cap \bar{A} \neq \varnothing$.

Then $\bar{A}=\mathcal{C}\left(T_{i}\right)$.

The proof of Proposition A.1 is step by step the same as the proof of Theorem 1 in McAfee and Reny (1992), except that the unit interval as the domain of functions must be 
replaced by $T_{i}$ and intervals of the form $\left[t_{i 0}-\delta, t_{i 0}+\delta\right]$ must be replaced by the closed $\delta$-balls $B^{\delta}\left(t_{i 0}\right)$ around $t_{i 0}$.

Similarly, the proof of the necessity of condition $\left(^{*}\right)$ for full surplus extraction is step by step the same as the proof of the necessity statement in Theorem 2 of McAfee and Reny, again with the proviso that the interval $[0,1]$ be replaced by $T_{i}$ and intervals of the form $\left[t_{i 0}-\delta, t_{i 0}+\delta\right]$ be replaced by the closed $\delta$-balls $B^{\delta}\left(t_{i 0}\right)$ around $t_{i 0}$.

Before turning to the proof of sufficiency of condition $\left(^{*}\right)$ for full surplus extraction, we recall the following notation from McAfee and Reny. Given a belief function $b_{i}$, we set

$$
R\left(b_{i}\right):=\left\{y: T_{i} \rightarrow \mathbb{R} \mid y\left(t_{i}\right)=\int z\left(t_{-i}\right) b_{i}\left(d t_{-i} \mid t_{i}\right) \text { for all } t_{i}, \text { for some } z \in C\left(T_{-i}\right)\right\}
$$

and

$r\left(b_{i}\right):=\left\{x: T_{i} \rightarrow \mathbb{R} \mid x\left(t_{i}\right)=\min \left\{y_{1}\left(t_{i}\right), \ldots, y_{N}\left(t_{i}\right)\right\}\right.$ for all $t_{i}$, for some $N$ and $y_{1}, \ldots, y_{N}$ in $R\left(b_{i}\right)$.

Under the given assumptions on $b_{i}, T_{i}$, and $T_{-i}$, with a topology on $\mathcal{M}\left(T_{-i}\right)$ that is at least as fine as the weak* topology, we have

$$
R\left(b_{i}\right) \subset C\left(T_{i}\right) \text { and } r\left(b_{i}\right) \subset C\left(T_{i}\right) .
$$

\section{Proof of the sufficiency statement in Theorem 2.1.}

As in McAfee and Reny (1992), the proof is indirect. Suppose condition (*) is not sufficient for full surplus extraction. Then there exists $b_{i} \in \mathcal{C}\left(T_{i}, \mathcal{M}\left(T_{-i}\right)\right)$ such that $\left(^{*}\right)$ holds for all $\bar{t}_{i} \in T_{i}$ and all $\mu \in \mathcal{M}\left(T_{i}\right)$ and $\mathcal{C}\left(T_{i}\right) \backslash \bar{r}\left(b_{i}\right) \neq \varnothing$, where $\bar{r}\left(b_{i}\right)$ is the closure of $r\left(b_{i}\right)$. By Proposition A.1, $\mathcal{C}\left(T_{i}\right) \backslash \bar{r}\left(b_{i}\right) \neq \varnothing$ implies that the set $r\left(b_{i}\right)$ violates one of the conditions in that proposition; the only candidate is condition (iv). Thus there exist $\varepsilon_{0}>0, \delta_{0}>0$, and $t_{i 0} \in T_{i}$ such that $U\left(\varepsilon_{0}, \delta_{0}, t_{i 0}\right) \cap \bar{r}\left(b_{i}\right)=\varnothing$. Since $R\left(b_{i}\right) \subset r\left(b_{i}\right)$, it follows that $U\left(\varepsilon_{0}, \delta_{0}, t_{i 0}\right) \cap \bar{R}\left(b_{i}\right)=\varnothing$, where $\bar{R}\left(b_{i}\right)$ is the closure of $R\left(b_{i}\right)$.

As discussed by McAfee and Reny (1992), one can now use the separating hyperplane theorem and the Riesz representation theorem to assert the existence of a constant $c$ and a regular, countably additive, signed measure $\mu \neq 0$ on $T_{i}$ such that

$$
\begin{aligned}
& \int_{T_{i}} x\left(t_{i}\right) d \mu\left(t_{i}\right) \leq c \text { for all } x \in \bar{R}\left(b_{i}\right), \text { and } \\
& \int_{T_{i}} x\left(t_{i}\right) d \mu\left(t_{i}\right) \geq c \text { for all } x \in U\left(\varepsilon_{0}, \delta_{0}, t_{i 0}\right) .
\end{aligned}
$$


Since $\bar{R}\left(b_{i}\right)$ is a linear subspace of $\mathcal{C}\left(T_{i}\right)$, the constant $c$ must actually be zero and we must have

$$
\int_{T_{i}} x\left(t_{i}\right) d \mu\left(t_{i}\right)=0 \text { for all } x \in \bar{R}\left(b_{i}\right),
$$

By the definition of $R\left(b_{i}\right)$, it follows that

$$
\int_{T_{i}} \int_{T_{-i}} z\left(t_{-i}\right) d b_{i}\left(t_{-i} \mid t_{i}\right) d \mu\left(t_{i}\right)=0
$$

for all $z \in \mathcal{C}\left(T_{-i}\right)$.

By the Jordan decomposition theorem, we may write $\mu$ as the difference between two positive measures $\mu^{+}$and $\mu^{-}$, at least one of which is finite. Thus, (53) can be rewritten in the form

$$
\int_{T_{i}} \int_{T_{-i}} z\left(t_{-i}\right) d b_{i}\left(t_{-i} \mid t_{i}\right) d \mu^{+}\left(t_{i}\right)=\int_{T_{i}} \int_{T_{-i}} z\left(t_{-i}\right) d b_{i}\left(t_{-i} \mid t_{i}\right) d \mu^{-}\left(t_{i}\right) .
$$

If $z \in \mathcal{C}\left(T_{-i}\right)$ is the constant function with value one, (54) specializes to the equation

$$
\mu^{+}\left(T_{i}\right)=\mu^{-}\left(T_{i}\right)
$$

so $\mu^{+}$and $\mu^{-}$are both finite. Moreover, there is no loss of generality in setting $\mu^{+}\left(T_{i}\right)=$ $\mu^{-}\left(T_{i}\right)=1$, so both $\mu^{+}$and $\mu^{-}$belong to $\mathcal{M}\left(T_{i}\right)$. (54) can therefore be rewritten in the form

$$
\int_{T_{-i}} z\left(t_{-i}\right) d \varphi_{b_{i}}\left(t_{-i} \mid \mu^{+}\right)=\int_{T_{-i}} z\left(t_{-i}\right) d \varphi_{b_{i}}\left(t_{-i} \mid \mu^{-}\right) .
$$

If (55) is to hold for all $z \in \mathcal{C}\left(T_{-i}\right)$, it must be the case that

$$
\varphi_{b_{i}}\left(\mu^{+}\right)=\varphi_{b_{i}}\left(\mu^{-}\right) .
$$

By $\left(^{*}\right)$, it follows that neither $\mu^{+}$nor $\mu^{-}$can be the point measure $\delta_{t_{i 0}}$ at $t_{i 0}$. For suppose that $\mu^{+}=\delta_{t_{i 0}}$. Then (56) implies $\varphi_{b_{i}}\left(\mu^{-}\right)=\varphi_{b_{i}}\left(\delta_{t_{i 0}}\right)$ and, by $\left(^{*}\right), \mu^{-}=\delta_{t_{i 0}}$, which is incompatible with $\mu=\mu^{+}-\mu^{-} \neq 0$. By a precisely symmetric argument, $\mu^{-}=\delta_{t_{i 0}}$ is also impossible.

Since $\mu^{-}$is regular, it follows that there exists a closed set $A \subset T_{i}$, such that $\mu^{-}(A)>$ $0, \mu^{+}(A)=0$, and for some $\delta \in\left(0, \delta_{0}\right]$, the intersection of $A$ with the $\delta$-ball $B^{\delta}\left(t_{i 0}\right)$ around $t_{i 0}$ is empty. Fixing $K>1 / \mu^{-}(A)$, we can define a step function $x^{K}$ by setting

$$
\begin{aligned}
& x^{K}\left(t_{i}\right)=0 \text { if } t_{i} \in B^{\delta}\left(t_{i 0}\right), \\
& x^{K}\left(t_{i}\right)=K \text { if } t_{i} \in A, \\
& x^{K}\left(t_{i}\right)=1 \text { otherwise. }
\end{aligned}
$$


For this step function, we find $\int x^{K}\left(t_{i}\right) d \mu\left(t_{i}\right) \leq 1-K \mu^{-}(A)<0$. Now $x^{K}$ itself is not continuous, but can be approximated by a sequence $\left\{x_{n}\right\}_{n=1}^{\infty}$ of continuous functions so that the integrals $\int x_{n}\left(t_{i}\right) d \mu\left(t_{i}\right)$ converge to $\int x^{K}\left(t_{i}\right) d \mu\left(t_{i}\right)$ as $n$ becomes large. For any sufficiently large $n$, therefore, $\int x_{n}\left(t_{i}\right) d \mu\left(t_{i}\right)<0$.

However, as discussed by McAfee and Reny (1992), the sequence $\left\{x_{n}\right\}_{n=1}^{\infty}$ can be chosen so that $x_{n} \in U\left(\varepsilon_{0}, \delta_{0}, t_{i 0}\right)$. By (51) and the fact that $c=0$, it follows that $\int x_{n}\left(t_{i}\right) d \mu\left(t_{i}\right) \geq$ 0 for all $n$. The assumption that condition $\left(^{*}\right)$ is not sufficient for full surplus extraction has thus led to a contradiction and must be false.

\section{Appendix B Necessity of infinite-dimensionality of the range in Theorem 2.4}

In more abstract terms, Theorem 2.4 can be restated as follows:

THEOREM B.1 Let $X$ and $Y$ be nonempty compact metric spaces. Assume that the topology on $\mathcal{M}(Y)$ is induced by a metric that is a convex function on $\mathcal{M}(Y) \times \mathcal{M}(Y)$. Let $\mathcal{E}^{*}\left(X_{i}, \mathcal{M}(Y)\right)$ be the set of continuous functions $b$ from $X$ to $\mathcal{M}(Y)$ that satisfy condition $\left(^{*}\right)$, i.e., the requirement that, for any $x \in X$ and any $\mu \in \mathcal{M}(X)$,

$$
\varphi_{b}(\mu)=\varphi_{b}\left(\delta_{x}\right) \text { implies } \mu=\delta_{x}
$$

where $\delta_{x}$ is the degenerate measure that assigns all mass to the singleton $\{x\}$ and $\varphi_{b}: \mathcal{M}(X) \rightarrow$ $\mathcal{M}(Y)$ is defined so that, for any $\mu \in \mathcal{M}(X)$

$$
\varphi_{b}(B \mid \mu)=\int b(B \mid x) \mu(d x)
$$

for all measurable $B \subset Y$. If $Y$ has more than finitely many elements, then $\mathcal{E}^{*}\left(X_{i}, \mathcal{M}(Y)\right)$ is a residual subset of the space $\mathcal{C}(X, \mathcal{M}(Y))$, i.e., $\mathcal{E}^{*}(X, \mathcal{M}(Y))$ contains a countable intersection of open and dense subsets of $\mathcal{C}(X, \mathcal{M}(Y))$.

In Section 2.5, we asserted that the genericity claim made in this theorem would be false if the functions under considerations have a finite-dimensional range. This contrasts with the genericity of embeddings, which by the classical embedding theorem holds whenever the dimension of the range is more than twice the dimension of the domain of the functions under consideration. The following result provides a formal statement. 
Proposition B. 2 Let $X$ be a metric space with more then $N$ elements. Let $C\left(X,[0,1]^{N}\right)$ be the space of continuous functions from $X$ to $[0,1]^{N}$, endowed with the uniform topology. There exists an open subset $\mathcal{U}$ of $\mathcal{C}\left(X,[0,1]^{N}\right)$ such that, for every $f \in \mathcal{U}$, there exists $x \in X$ such that

$$
f(x) \in \operatorname{co}\{f(X \backslash\{x\})\},
$$

i.e., the value of the function at $x$ belongs to the convex hull of its values at points other than $x$.

Corollary B.3 For $X$ as specified in Proposition B.2, let $\mathcal{E}^{*}\left(X,[0,1]^{N}\right)$ be the subset of those functions $f \in \mathcal{C}\left(X,[0,1]^{N}\right)$ for which there exists no $x \in X$ for which (57) holds. Then the set $\mathcal{E}^{*}\left(X,[0,1]^{N}\right)$ is not dense in $\mathcal{C}\left(X,[0,1]^{N}\right)$.

Proof of Proposition B.2. Choose $p_{1}, \ldots, p_{N+2}$ in $[0,1]^{N}$ so that $p_{1}, \ldots, p_{N+1}$ are in general position $^{33}$ and

$$
p_{N+2}=\sum_{i=1}^{N+1} \alpha_{i} p_{i}
$$

where $\sum_{i=1}^{N+1} \alpha_{i}=1$ and $\alpha_{i}>0$ for all $i$.

Fix a function $g \in C\left(X,[0,1]^{N}\right)$ such that, for $i=1, \ldots, N+2$,

$$
g\left(x_{i}\right)=p_{i}
$$

Then, obviously,

$$
g\left(x_{N+2}\right) \in \operatorname{co}\left\{g\left(x_{1}\right), \ldots, g\left(x_{N+1}\right)\right\} ;
$$

In fact, since $\alpha_{i}>0$ for all $i, g\left(x_{N+2}\right)=p_{N+2}$ belongs to the interior of $\operatorname{co}\left\{g\left(x_{1}\right), \ldots, g\left(x_{N+1}\right)\right\}$, i.e., there exists $\varepsilon>0$ such that the $\varepsilon$-ball $B^{\varepsilon}\left(p_{N+2}\right)$ around $g\left(x_{N+2}\right)=p_{N+2}$ is fully contained in the convex hull of $g\left(x_{1}\right)=p_{1}, \ldots, g\left(x_{N+1}\right)=p_{N+1}$.

Now, fix $\eta=\frac{\varepsilon}{2}$ and let $\mathcal{U}$ be the open $\eta$-neighbourhood of $g$, i.e. the set of all functions $f$ such that $d(f(x), g(x))<\eta$ for all $x \in X$, where $d$ is the metric on $[0,1]^{N}$. We claim that, for any $f \in \mathcal{U}$,

$$
f\left(x_{N+2}\right) \in \operatorname{co}\left\{f\left(x_{1}\right), \ldots, f\left(x_{N+1}\right)\right\} .
$$

To prove this claim, we will show that the $\frac{\varepsilon}{2}$-ball $B^{\frac{\varepsilon}{2}}\left(p_{N+2}\right)$ around $g\left(x_{N+2}\right)=p_{N+2}$ is a subset of the convex hull of $f\left(x_{1}\right), \ldots, f\left(x_{N+1}\right)$, i.e., that

$$
B^{\frac{\varepsilon}{2}}\left(p_{N+2}\right) \subset \operatorname{co}\left\{f\left(x_{1}\right), \ldots, f\left(x_{N+1}\right)\right\} .
$$

\footnotetext{
${ }^{33} r$ distinct vertices are in general position in $[0,1]^{N}$, if there are no $m+2$ vertices $(m=1, \ldots, N-1)$ among them that lie in an $m$-dimensional linear subspace of $[0,1]^{N}$.
} 
(62) implies (61) because, for $f \in \mathcal{U}, d\left(f\left(x_{N+2}\right), g\left(x_{N+2}\right)\right)<\eta=\frac{\varepsilon}{2}$, and therefore, $f\left(x_{N+2}\right) \in B^{\frac{\varepsilon}{2}}\left(p_{N+2}\right)$.

To prove (62), we first note that

$$
\operatorname{co}\left\{p_{1}, \ldots, p_{N+1}\right\} \subset B^{\eta}\left(\operatorname{co}\left\{f\left(x_{1}\right), \ldots, f\left(x_{N+1}\right)\right\}\right) .
$$

To see this, observe that, for any $q \in \operatorname{co}\left\{p_{1}, \ldots, p_{N+1}\right\}$, there exist $\alpha_{i}^{q}, i=1, \ldots, N+1$, such that

$$
q=\sum_{i=1}^{N+1} \alpha_{i}^{q} p_{i}
$$

For $f \in \mathcal{U}$, the distance between $q$ and the element $\sum_{i=1}^{N+1} \alpha_{i}^{q} f\left(x_{i}\right)$ of $\operatorname{co}\left\{f\left(x_{1}\right), \ldots, f\left(x_{N+1}\right)\right\}$ is less than $\eta$, i.e. $q \in B^{\eta}\left(\left\{\operatorname{co}\left\{f\left(x_{1}\right), \ldots, f\left(x_{N+1}\right)\right\}\right)\right.$.

Since $B^{\varepsilon}\left(p_{N+2}\right) \subset \operatorname{co}\left\{p_{1}, \ldots, p_{N+1}\right\}$, it follows that

$$
B^{\varepsilon}\left(p_{N+2}\right) \subset B^{\eta}\left(\operatorname{co}\left\{f\left(x_{1}\right), \ldots, f\left(x_{N+1}\right)\right\}\right) .
$$

Now (62) follows because $\eta=\frac{\varepsilon}{2}$. For suppose that (62) fails so that there exists some $v \in B^{\frac{\varepsilon}{2}}\left(p_{N+2}\right) \backslash \operatorname{co}\left\{f\left(x_{1}\right), \ldots, f\left(x_{N+1}\right)\right\}$. Let $y$ be the element of $c o\left\{f\left(x_{1}\right), \ldots, f\left(x_{N+1}\right)\right\}$ that is closest to $v$, and let

$$
z=v+\delta(v-y)
$$

where $\delta$ is chosen so that $d(z, v)$, the distance between $z$ and $v$, is exactly $\eta$. By the triangle inequality,

$$
d\left(p_{N+2}, z\right) \leq d\left(p_{N+2}, v\right)+d(v, z) .
$$

By construction, $d(v, z)=\eta=\frac{\varepsilon}{2}$ and $d\left(p_{N+2}, v\right) \leq \frac{\varepsilon}{2}$. Thus, $z \in B^{\varepsilon}\left(p_{N+2}\right)$. By (64), it follows that there exists $\left.u \in \operatorname{co}\left\{f\left(x_{1}\right), \ldots, f x_{N+1}\right)\right\}$ such that

$$
d(z, u) \leq \eta
$$

Using (65), we obtain

$$
d(z, u)=d(v+\delta(v-y), u)=(1+\delta) d(v, \lambda u+(1-\lambda) y),
$$

where $\lambda:=\frac{1}{1+\delta}$. By the definition of $y$ and another application of (65), it follows that

$$
d(z, u) \geq(1+\delta) d(v, y)>\delta d(v, y)=d(z, v)
$$

Upon combining (66) and (67), we find that $d(z, v)<\eta$, contrary to the assumption that $d(z, v)=\eta$. The assumption that (62) fails has thus led to a contradiction and must be false. 


\section{References}

Aliprantis, C. AND K. BORDER (2007): Infinite-Dimensional Analysis: A Hitchhiker's Guide, 3rd Edition, Springer.

BARELli, P. (2009): "On the genericity of full surplus extraction in mechanism design," $J$. of Econ. Theory, 144, 1320 - 1333.

BergemanN, D. AND S. MORRIS (2005): “Robust mechanism design,” Econometrica, 73, 1521-1534.

Chen, Y., A. DiTillio, E. Faingold, And S. XiOng (2010): “Uniform Topologies on Types," Theoretical Economics, 5, 445 - 478.

CHEN, Y. AND S. XIONG (2011): “The Genericity of Beliefs-Determine-Preferences Models Revisited," Journal of Economic Theory, 146, 751- 761.

- (2013): “Genericity and Robustness of Full Surplus Extraction," Econometrica, 81, $825-847$.

Chen, Y.-C., A. DiTillio, E. FAingold, And S. XiOng (2016): “Corrigendum: Uniform Topologies on Types," Working paper, Yale University.

CRÉMER, J. AND R. MCLEAN (1988): “Full extraction of the surplus in Bayesian and dominant strategy auctions," Econometrica, 56, 1247-1257.

Dekel, E., D. Fudenberg, And S. Morris (2006): “Topologies on Types," Theoretical Economics, 1, $275-309$.

DeKel, E. AND M. SinISCAlCHI (2015): Epistemic Game Theory, Chapter in Handbook of Game Theory with Economic Applications, Volume 4, Young, H. P. and Zamir, S.

Dudley, R. (2002): Real Analysis and Probability, Cambridge: Cambridge University Press.

DUNFORD, N. AND J. SCHWARTZ (1988): Linear Operators, General Theory, Wiley Interscience.

ENGELKING, R. (1989): General Topology, Berlin: Springer-Verlag.

ENGL, G. (1995): “Lower Hemicontinuity of the Nash Equilibirum Correspondence," Games and Economic Behaviour, 9, 151-160. 
Gizatulina, A. (2015): “Betting On Others' Bets: The Generalized Surplus Extraction Mechanism," Working paper, University of St. Gallen.

Gizatulina, A. And M. Hellwig (2013): “On the Designer Uncertainty and the Robustness of Full Surplus Extraction in Families of Incomplete-Information Models," Working paper, Max Planck Institute for Research on Collective Goods.

- (2014): "Beliefs, Payoffs, Information: On the Robustness of the BDP Property in Models with Endogenous Beliefs," Journal of Mathematical Economics, 51, 136-153.

GÜTH, W. AND M. HellWiG (1986): “The private supply of a public good," Journal of Economics, 5, 121-159.

Heifetz, A. AND Z. NeEman (2006): “On the generic (im)possibility of full surplus extraction in mechanism design," Econometrica, 117, 213-233.

Hellwig, M. (2017a): “A Homeomorphism Theorem for the Universal Type Space with the Uniform Weak Topology," Preprint 17/2016, revised, Max-Planck Institute for Research on Collective Goods.

(2017b): "Probability Measures on Product Spaces with a Uniform Metric," Preprint 06/2017, Max-Planck Institute for Research on Collective Goods.

Hurewicz, W. And H. Wallman (1941): Dimension Theory, Princeton, NJ: Princeton Mathematical Series, no. 4 .

Mailath, G. And A. Postlewaite (1990): “Asymmetric information bargaining problems with many agents," Rev. Econ. Stud., 57, 351-367.

MCAFEE, P. AND P. RENY (1992): “Correlated information and mechanism design," Econometrica, 60, 395-421.

MERTENS, J. AND S. ZAMIR (1985): “Formulation of Bayesian analysis for games with incomplete information," International Journal of Game Theory, 10, 619-632.

Naimpally, S. (1966): “Graph topology for function spaces,” Trans. Amer. Math. Soc., 123, 267-272.

NEEMAN, Z. (2004): “The relevance of private information in mechanism design," J. Econ. Theory, 117, 55-77. 
Parthasarathy, K. (1967): Probability Measures on Metric Spaces, New York and London: Academic Press.

RoB, R. (1989): "Pollution claim settlements under private information," Journal of Economic Theory, 47, $307-333$.

Rubinstein, A. (1989): “The Electronic Mail Game: Strategic Behavior under "Almost" Common Knowledge," American Economic Review, 79, 385-391. 